Check for updates

Cite this: RSC Adv., 2019, 9, 6460

\title{
Carbon quantum dots and their biomedical and therapeutic applications: a review
}

\begin{abstract}
Mohammad Jafar Molaei (iD *
In recent years, nano carbon quantum dots (CQDs) have received increasing attention due to their properties such as small size, fluorescence emission, chemical stability, water solubility, easy synthesis, and the possibility of functionalization. CQDs are fluorescent OD carbon nanostructures with sizes below $10 \mathrm{~nm}$. The fluorescence in CQDs originates from two sources, the fluorescence emission from bandgap transitions of conjugated $\pi$-domains and fluorescence from surface defects. The CQDs can emit fluorescence in the near-infrared (NIR) spectral region which makes them appropriate for biomedical applications. The fluorescence in these structures can be tuned with respect to the excitation wavelength. The CQDs have found applications in different areas such as biomedicine, photocatalysis, photosensors, solar energy conversion, light emitting diodes (LEDs), etc. The biomedical applications of CQDs include bioimaging, drug delivery, gene delivery, and cancer therapy. The fluorescent CQDs have low toxicity and other exceptional physicochemical properties in comparison to heavy metals semiconductor quantum dots (QDs) which make them superior candidates for biomedical applications. In this review, the synthesis routes and optical properties of the CQDs are clarified and recent advances in CQDs biomedical applications in bioimaging (in vivo and in vitro), drug delivery, cancer therapy, their potential to pass blood-brain barrier (BBB), and gene delivery are discussed.
\end{abstract}

Received 29th September 2018 Accepted 14th February 2019

DOI: $10.1039 / \mathrm{c} 8 \mathrm{ra0} 8088 \mathrm{~g}$

rsc.li/rsc-advances biocompatibility, up-conversion, tunable photoluminescence (PL), and chemical stability. ${ }^{\mathbf{1}, 2}$

The CQDs are small nanoparticles made of carbon which include different surface passivation by functionalization or modification. ${ }^{3}$ The CQDs can be crystalline or amorphous. The carbon hybridization in CQDs is $\mathrm{sp}^{2}$ while in some cases, $\mathrm{sp}^{3}$ hybridization has also been reported. ${ }^{1}$ The CQDs are categorized into $0 \mathrm{D}$ nanostructures and their sizes are mostly below $10 \mathrm{~nm}$. The crystal lattice parameter for CQD is about $0.34 \mathrm{~nm}$, corresponding to (002) graphite interlayer spacing. ${ }^{4}$

The CQDs could be functionalized with a variety of surface groups. Oxygen-containing functional groups (hydroxyl and carboxyl) are mostly present on the CQDs surface that result in their water solubility. ${ }^{4}$ The functional groups on the CQDs also result in solubility, forming stable colloids in aqueous or polar organic solvents which is advantageous over graphene quantum dots (GQDs) that have a low solubility in common solvents. ${ }^{5}$ The surface groups might impart fluorescence properties of the CQDs. ${ }^{4}$

The carbon nanostructures that have fluorescence properties are versatile and include GQDs, ${ }^{6-8}$ carbon nanotube quantum dots (CNT QDs), ${ }^{9}$ graphene oxide (GO), ${ }^{\mathbf{1 0}, 11}$ nanodiamonds $(\mathrm{NDs})^{12,13}$ and CQDs. ${ }^{14}$

Carbon dots (CDs) refers to the structures with at least one dimension below $10 \mathrm{~nm}$ that have PL properties. The CDs have oxygen/nitrogen-containing functional groups or polymeric aggregations. Mainly, the CDs include GQDs, polymer dots (PDs) and CQDs. ${ }^{15}$ 
GQDs are tiny fragments of graphene sheet in them, the electronic transport is confined in all 3D dimensions. Graphene is a semiconductor with zero bandgap that its exciton Bohr diameter is infinite. Therefore, any fragment of graphene experiences confinement. However, the GQDs refers to graphene fragments that have a size below $20 \mathrm{~nm}$. The GQDs can be fabricated via cutting of the graphene sheet. ${ }^{16}$ The quantum confinement and edge effects of the GQDs induce PL. ${ }^{17}$ The GQDs have a non-zero bandgap and therefore, can show PL if are excited. The GQDs bandgap can be tuned with controlling the size and surface chemistry. ${ }^{16}$ The PDs consist of $\pi$-conjugated polymers that in small particle sizes show brightness. The PDs have versatile applications including fluorescence imaging. ${ }^{18}$ Table 1 summarizes different fluorescent carbon nanostructures, their structure, and fluorescence mechanisms.

Here in this paper, we exclude GQDs and PDs from CDs and the discussions are mainly on the CQDs. The synthesis and optical properties of CQDs and the fluorescence mechanisms are expressed and recent advances in their biomedical applications are discussed.

\section{Synthesis}

Sun et al. first reported the production of fluorescent CQDs through laser ablation. The laser ablation was conducted on a carbon target while argon was used as carrier gas. The synthesized CQDs were passivated with PEG1500N species. ${ }^{\mathbf{1 4}}$ There are several synthesize methods for production of CQDs that are categorized in top-down and bottom-up routes. The topdown route means breaking apart of a larger bulk material/ nanomaterial down to the particle sizes below $10 \mathrm{~nm}$. The methods such as laser ablation, ${ }^{23-25}$ electrochemical ${ }^{26,27}$ and high energy ball milling ${ }^{28}$ are categorized under the top-down methods for synthesis of CQDs. The bottom-up methods for synthesizing of CQDs which means synthesis from small molecules or carbonization, include hydrothermal, ${ }^{29-31}$ solvothermal, ${ }^{32,33}$ combustion, ${ }^{34}$ pyrolysis, ${ }^{35}$ microwave-assisted synthesis, ${ }^{36-41}$ ultrasonic, ${ }^{\mathbf{4 2 - 4 4}}$ reverse micelles, ${ }^{\mathbf{4 5 , 4 6}}$ chemical vapor deposition (CVD), ${ }^{47}$ and etc. Table 2 summarizes different synthesis methods of CQDs from versatile precursors, their optical properties, and their applications.

Fig. 1 and 2 show some of the synthesis methods of CQDs from different precursors.

Fig. 1a shows the synthesis method of CQDs that were hydrothermally achieved from slices of carrots after heating in an autoclave at $170{ }^{\circ} \mathrm{C}$ for $12 \mathrm{~h} .{ }^{78} \mathrm{Fig}$. $1 \mathrm{~b}$ shows that CQDs can be obtained from pyrolytic residue (WP) chemical oxidation with a mixture of $\mathrm{HNO}_{3}$ and $\mathrm{H}_{2} \mathrm{SO}_{4}$ in presence of ultrasonic waves followed by heat treatment in an autoclave at $120{ }^{\circ} \mathrm{C}$ for $12 \mathrm{~h}^{79}$

Fig. 1c shows the synthesis of nitrogen-rich CQDs by adding oleylamine to polyacrylamide (PAA) solution (50 $\mathrm{wt} \%$ in water) and nitric acid. The mixture was stirred at $50{ }^{\circ} \mathrm{C}$ under argon atmosphere to form a milky emulsion. The opaque state of the solution indicates that the water droplet sizes in the solution are in the micrometer range. Then the solution was heated to $250{ }^{\circ} \mathrm{C}$ under the same atmosphere. The heat evaporates the water, and water droplet sizes reach to the nanometer range. Water evaporation makes the emulsion to supersaturate and results in carbonization of polyacrylamide chains while oleylamine plays as both oil phase solvent and capping agent. The solution was precipitated with methanol. ${ }^{80}$ Fig. 2a shows a schematic of the CVD synthesis method of the CQDs using $\mathrm{C}_{2} \mathrm{H}_{2}$ as the carbon source. A mixture of the $\mathrm{C}_{2} \mathrm{H}_{2}$ and Ar gas are introduced in a quartz tube at $1000{ }^{\circ} \mathrm{C}$. The deposited product was dissolved in $N, N$-dimethylformamide and was filtered to remove undesirable particles. ${ }^{47}$ Fig. $2 \mathrm{~b}$ is an illustration of the hydrothermal synthesis method of the N-doped CQDs using alanine and ethylenediamine as the carbon source and surface

Table 1 Different fluorescent carbon nanostructures, their structure and fluorescence mechanisms

\begin{tabular}{lll}
\hline Fluorescent carbon nanostructures & Structure & Optical properties
\end{tabular}

CNT dots

Graphene materials, GQDs

Nanodiamonds

PDs

Fullerenes

CQDs
Short CNTs with $\mathrm{sp}^{2}$ hybridization

As-synthesized GO thin films, mechanically exfoliated, oxygen plasma-treated, single-layer graphene, reduced GO (rGO), chemically modified GO or rGO, fragments of graphene sheets $^{20}$

Nanosized diamond powders

PDs are $\pi$-conjugated polymers and aggregated or cross-linked polymers which are synthesized from monomers or linear polymers, as well as self-assembled polymer chains on carbon core $^{15}$ Buckyball shape carbon allotropes such as C60 and C70

Amorphous or crystalline carbon dots typically less than $10 \mathrm{~nm}$ in size
The PL mechanisms are bandgap fluorescence and PL due to the defects ${ }^{19}$

PL from defects and from bandgap transitions correlated to the conjugated $\pi$-domains are the main suggested PL mechanisms ${ }^{19}$

Optically transparent and capable of fluorescent emission from nitrogen-vacancy center defects with negative charge ${ }^{12}$

The PL mechanism of PDs is crosslinkenhanced emission (CEE) effect ${ }^{15}$

Unlike nonfluorescent $\mathrm{C} 60$, the $\mathrm{C} 70$ could generate normal and delayed fluorescence. The fluorescence quenches by oxygen and is influenced by temperature ${ }^{21}$

The fluorescence mechanisms are PL correlated to surface defects and the PL correlated to conjugated $\pi$-domains ${ }^{22}$ 
Table 2 Different synthesis routes of CQDs from different precursors, their optical properties and applications

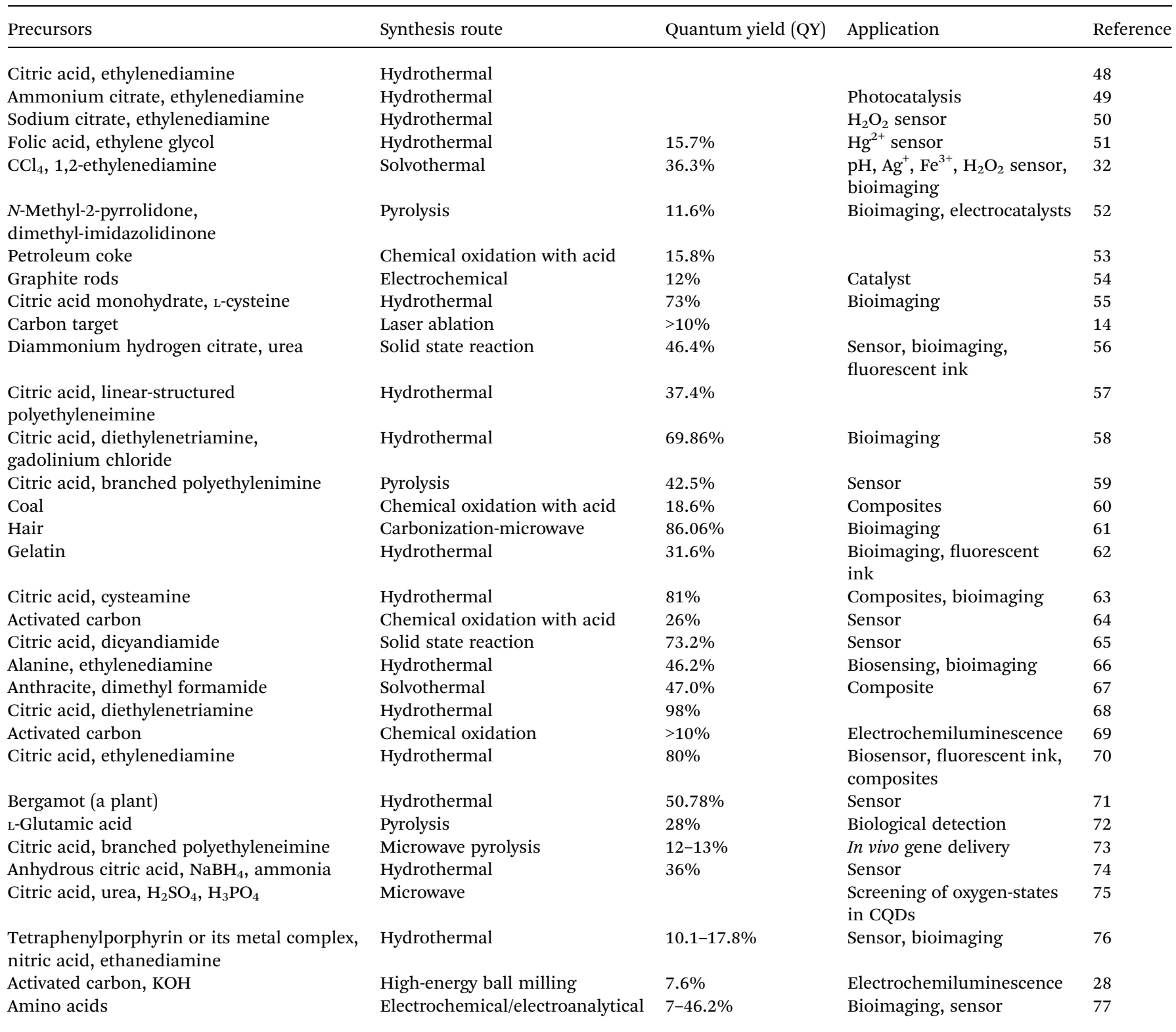

passivation agent, respectively. ${ }^{66}$ Fig. $2 \mathrm{c}$ is another schematic illustration that shows microwave-assisted pyrolysis synthesis of magnetofluorescent CQDs from crab shell as the carbon source and as a chelating ligand to result in complexes with $\mathrm{Gd}^{3+}, \mathrm{Mn}^{2+}$, and $\mathrm{Eu}^{3+}$. By application of transition-metal ions, CQDs with excellent magnetic resonance response were synthesized. ${ }^{81}$

\section{Optical properties}

The fluorescence in CQDs can originate from two sources: (i) fluorescence emission from bandgap transitions of conjugated $\pi$-domains and (ii) fluorescence form surface defects., ${ }^{219}$ In a graphene sheet, a bandgap can be created if the infinite $\pi$ network convert to finite. Isolation of conjugated $\pi$-domains can be through the formation of $\mathrm{sp}^{2}$ islands in a graphene sheet or it can be through the cutting of the graphene sheet into small pieces or other similar ways. Reduction of exfoliated GO can form isolated $\mathrm{sp}^{2}$ domains on the graphene sheet. Through this way, the isolated $\mathrm{sp}^{2}$ islands can be surrounded by an $\mathrm{sp}^{3}$ matrix of oxygen and carbon, like immersing large aromatic molecules in non-conjugated polymers. The electron-hole pairs can be localized in the conjugated $\pi$-domains. The bandgap of the domains depends on their sizes. ${ }^{19}$

The fluorescence can be originated from surface defects as well. The surface defects are sites that miss having a perfect $\mathrm{sp}^{2}$ domain. These non-perfect $\mathrm{sp}^{2}$ domains act as surface energy traps. The functionalized surface defects and hybridized $\left(\mathrm{sp}^{2}\right.$ and $\mathrm{sp}^{3}$ ) carbons can cause this type of fluorescence. The surface defects can be considered as aromatic molecules in a solid matrix. Since often there are multiple surface defects that each has its own excitation and emission wavelengths, the 
$\mathbf{a}$

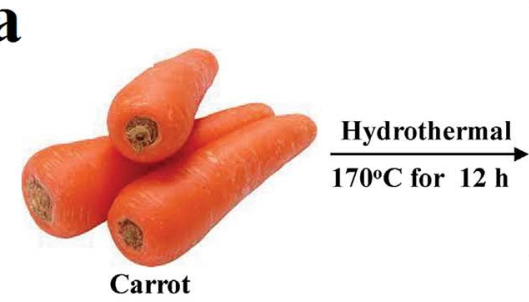

b

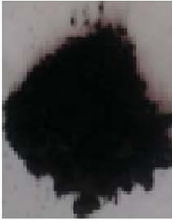

WP

residue

c

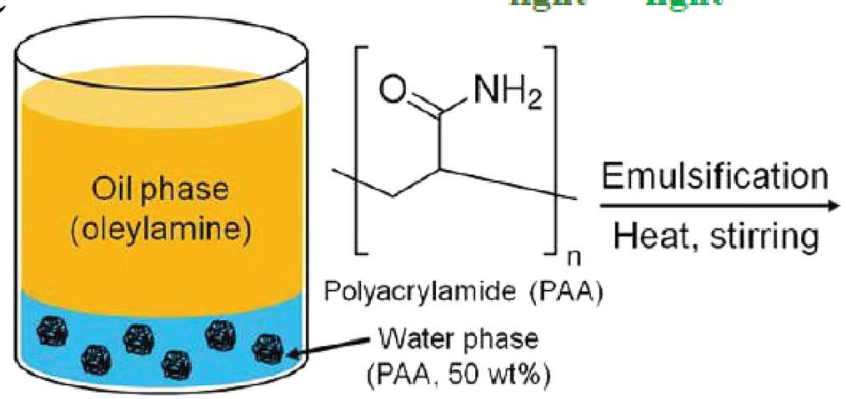

After addition $\rightarrow$

of $\mathrm{Cu}^{2+}$

ions

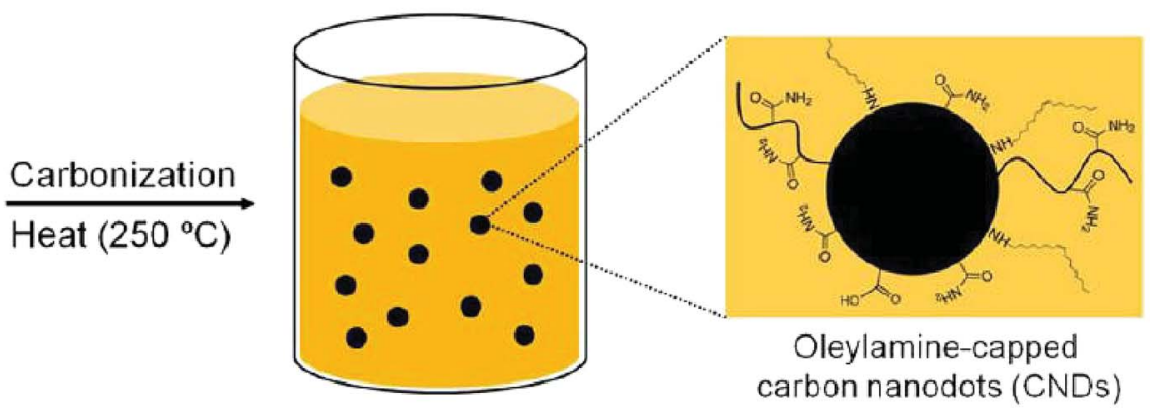

Fig. 1 (a) Synthesis route of CQDs from carbon source of carrots through hydrothermal method, reprinted with permission from ref. 78, copyright 2018 Elsevier Ltd., (b) synthesis of CQDs from the pyrolytic residue by chemical oxidation, when the $\mathrm{Cu}^{2+}$ ions are present in the solution, the fluorescence quenches, reprinted with permission from ref. 79, copyright 2018 Elsevier Ltd., (c) synthesis of CQDs from carbonization of polyacrylamide chains by formation a microemulsion. Reprinted with permission from ref. 80 , copyright 2013 American Chemical Society.

total emission can be multi-color. Recombination of electronhole pairs in the localized $\pi$ and $\pi^{*}$ levels of sp $^{2}$ domains leads to fluorescence emission..$^{22}$

\section{Biomedical applications}

The CQDs possess special physicochemical and catalytic properties that can make them as candidates for some biomedical applications. Two characteristics of CQDs i.e. their small size, as well as biocompatibility, can give them great opportunity to be used as drug delivery vehicles while can be monitored in the body due to their PL characteristics. ${ }^{1}$ The CQDs with minor toxicity, high hydrophilicity and water solubility, excitationwavelength-dependent PL emission, and chemical stability have versatile potential applications in the biomedical fields. Hereafter, we focus on the main bio-applications of the CQDs and recent advances in each area will be discussed.

\subsection{Optical imaging}

Nanoparticles can be conjugated with peptides, antibodies or small molecules and can be used for detection of cancer cells and molecular biomarkers. Among them, there are iron oxides for MRI and drug delivery, nanoparticles for drug delivery, and 
$\mathbf{a}$

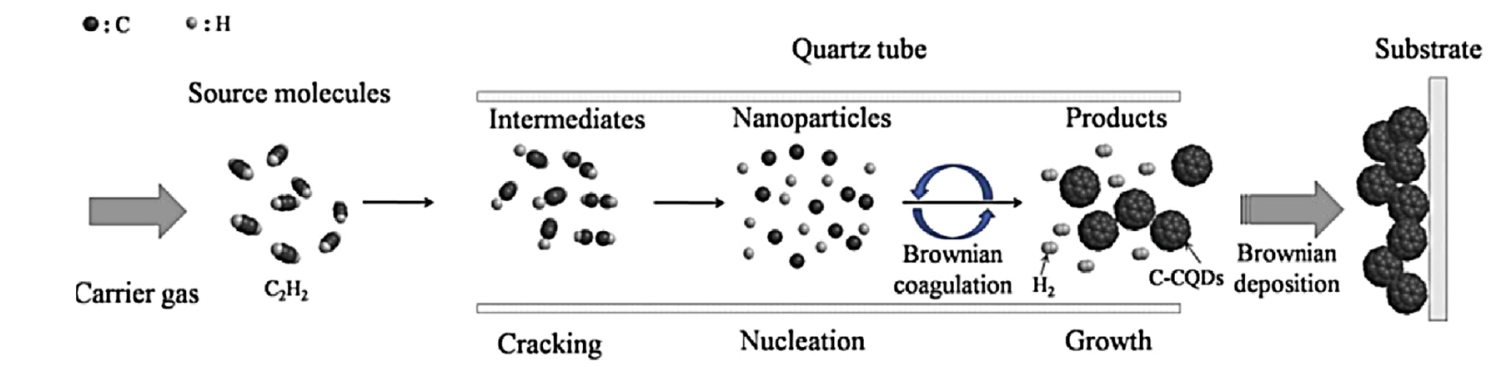

b
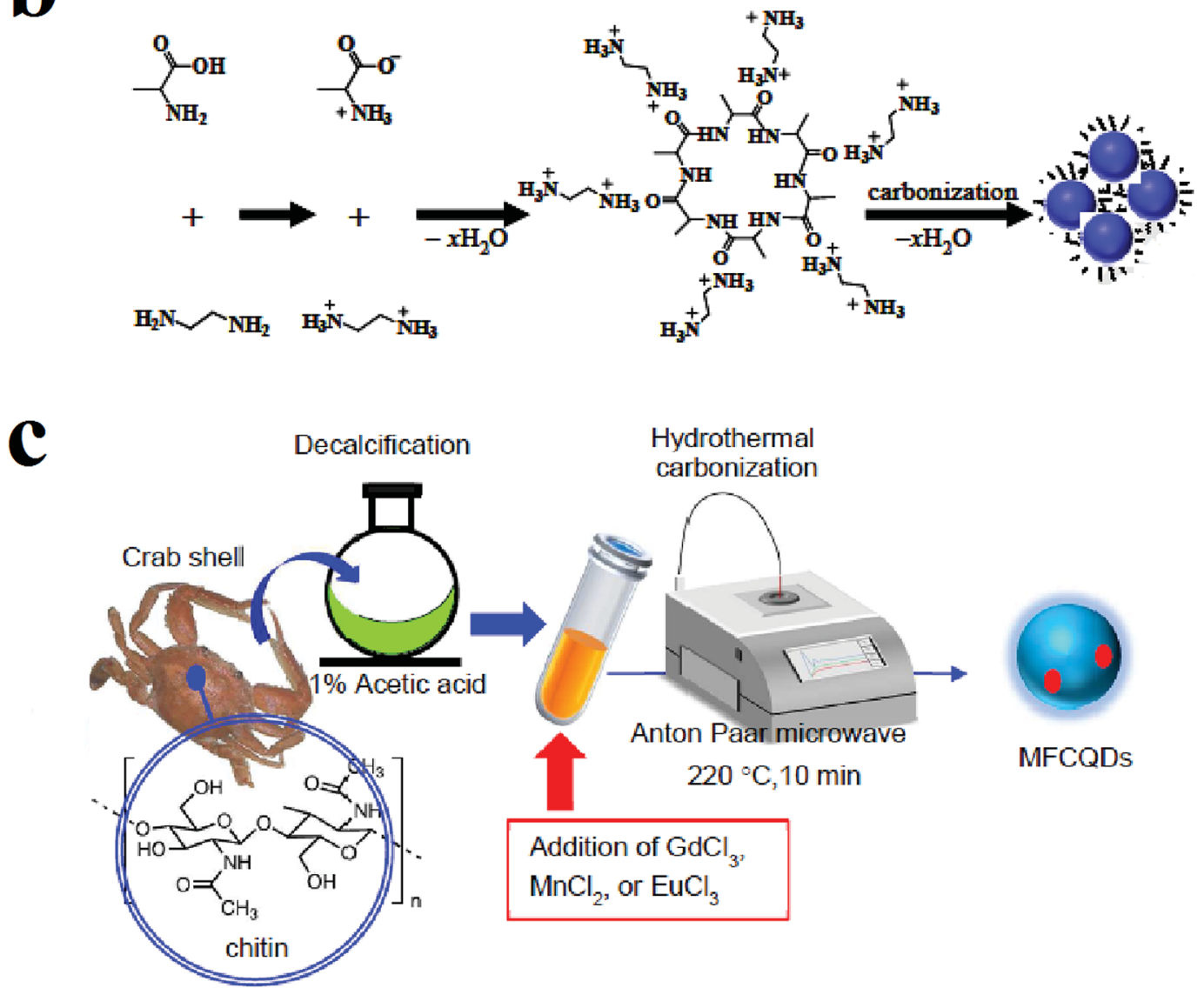

Fig. 2 (a) Synthesis of CQDs through CVD method using $\mathrm{C}_{2} \mathrm{H}_{2}$ as the carbon source, reprinted with permission from ref. 47, copyright 2016 Elsevier Ltd., (b) synthesis of the CQDs through the hydrothermal method using alanine and ethylenediamine, reprinted with permission from ref. 66, copyright 2015 Elsevier Ltd., (c) microwave-assisted pyrolysis synthesis of magnetofluorescent CQDs from crab shell, reprinted with permission from ref. 81, copyright 2017 American Chemical Society.

QDs for bioimaging and molecular diagnosis. ${ }^{82}$ Moreover, single particle tracking (SPT) is a technique for monitoring of dynamic processes in biological systems. SPT can track single molecule behavior and hence, statistical characterization of the system can be done. ${ }^{83}$

The QDs with optical properties such as broad absorption and narrow emission spectra and high quantum yield (QY) have been extensively studied for application as fluorophores in bioimaging field. The quantum confinement effect in QDs leads to tunable luminescence with respect to their sizes. Briefly, the electron-hole pair in the bulk semiconductor has a characteristic distance that is called exciton Bohr radius. ${ }^{84}$ The quantum confinement puts a limitation on the number of the possible energy states of an electron. ${ }^{85}$ By decreasing the size and reaching to sizes below exciton Bohr radius, the electron energy levels become discrete and the bandgap increases. Therefore, the QDs with a constant chemical composition can display fluorescence emissions with different wavelengths, if their sizes are different. ${ }^{84}$

The semiconductor QDs can be used for bioimaging, biodiagnostics, and they can be used as imaging probes for in vitro/ in vivo studies. Furthermore, multiplexed conjugates of QD- 
antibody can be used for molecular and cellular mapping of the human cancer specimens. ${ }^{82}$ The QDs can also be used for SPT at the level of the cell membrane, intracellular and on the brain slices. ${ }^{83}$ The QDs such as CdS, ${ }^{86-89} \mathrm{CdSe},{ }^{90,91} \mathrm{CdTe},{ }^{92,93} \mathrm{PbS},{ }^{94}$ and $\mathrm{MoS}_{2}$ (ref. 95-99) have been used for optical bioimaging. In QDS made of semiconductor materials, the inorganic core size is in the range of 4-10 nm. However, for biocompatibility, functionalizing, and stabilization of the QDs, in most cases, it is necessary that QDs be covered with additional shells or coatings that increase their sizes to $10-30 \mathrm{~nm}$. Increased size with regard to biocompatibility, and controlling the number of the sites for binding on the shell is challenging. Therefore, reducing the final size of the QDs and bio-conjugation strategies is still the matter of many types of research in this field. ${ }^{83}$ Furthermore, at the single-molecule level, there is a fluctuation in the emission of the fluorophores which is called blinking. This limits the biological applications of fluorophores at the single molecule level. The semiconductor QDs also have the drawback of blinking. ${ }^{100}$

The CQDs with the advantages of biocompatibility, nontoxicity, physicochemical stability, water solubility, and nonblinking characteristics can be considered as superior candidates for cell and tissue imaging. More explore on unconventional approaches might lead to the synthesis of the CQDs with fluorescence in the red and NIR regions of the light spectrum that are important in tissue penetration and decreased interference with the background fluorescence. ${ }^{3}$

4.1.1. In vitro imaging. Several researchers have successfully used the CQDs for in vitro bioimaging. ${ }^{.2,101-113}$ In several applications, the QDs are preferred to organic dyes and fluorescent proteins due to the enhanced brightness, photostability, imaging sensitivity, and good resistance to metabolic degradation. ${ }^{114}$ Semiconductor QDs such as CdSe with their core-shell structure (e.g. CdSe/ZnS) have been used for in vivo/in vitro bioimaging. ${ }^{115-121}$ However, the cadmium-containing QDS toxicity is the main concern due to their accumulation in tissues and organs. The CQDs that were synthesized through nitric acid treatment of carbon soot and subsequent high-temperature treatment with PEG1500N to be passivated with oligomeric PEG, were compared with classical semiconductor QDs (CdSe/ $\mathrm{ZnS}$ ) for cytotoxicity and bioimaging. It was found that the CQDs are biocompatible and are competitive in bioimaging in comparison with CdSe/ZnS QDs. Fig. 3 shows the fluorescence images of CQDs and CdSe/ZnS commercial QDs in the infinite dilution conditions. It can be seen that compared to commercial QDs, the CQDs are strongly fluorescent (QY of $20 \%$ by an excitation wavelength of $440 \mathrm{~nm}$ ) and non-blinking. The CQDs samples have a lower level of homogeneity which in turn makes it possible to individual brighter dots be isolated for enhanced optical properties. The CQDs have the advantage of smaller sizes compared to the CdSe/ZnS commercial QDs. The CQDs sizes are below $10 \mathrm{~nm}$ while the commercial QDs are larger than $20 \mathrm{~nm}$. The smaller sizes of CQDs provide the possibility of probing small biological structures and a minimal volume of in vivo injections. ${ }^{\mathbf{1 1 4}}$

Cao et al. synthesized CQDs with strong luminescence with two-photon excitation in the NIR region and used poly(propionyl ethylenimine-co-ethylenimine) for their surface passivation. They claimed that the two-photon absorption cross-sections of the synthesized and passivated CQDs were comparable with those of semiconductor QDs. The CQDs were water soluble with the sizes below $5 \mathrm{~nm}$. The luminescence intensities in the CQDs was dependent on the power of the excitation laser (pulsed infrared laser) that is evidence of the two-photon excitation. By incubation of human breast cancer cells (MCF-7) with the CQDs and excitation with $800 \mathrm{~nm}$ laser pulses, the cells were illuminated under the fluorescence microscope. The CQDs labeled cells membrane and cytoplasm while missed reaching to the nucleus. The CQDs accumulation in the cell would be enhanced if the CQDs be coupled with TAT (a human immunodeficiency virus-derived protein). By coupling with TAT, it would be possible for CQDs to reach the cell nucleus. $^{23}$

The surface passivation of CQDs for bioimaging can alter their optical properties. The CQDs were produced by the microwave treatment of maltose and passivated with diluted $\mathrm{NaOH}$ solution. The $\mathrm{NaOH}$ solution treatment causes attachment of $-\mathrm{OH}$ groups on the $\mathrm{sp}^{2}$ hybridized carbons that results in increasing of the CQDS QY. It was observed that the CQDS entered the cells and emitted green emission of fluorescence while cell viability was unaffected even after $24 \mathrm{~h}$ incubation with CQDs. ${ }^{36}$ In other experiments ${ }^{122}$ it was observed that surface passivation alters both fluorescence intensity and emission wavelength.

Functionalization is not a necessary step in the synthesis of CQDs if they are used as a labeling agent in bioimaging. However, the existence of $-\mathrm{OH}$ groups can make hydrophilic CQDs that is an advantage for bioimaging applications. For example, the CQDs with the particle size in the range of 2-6 $\mathrm{nm}$ and QY of 3\% were synthesized by soot reflux with nitric acid. The oxidation of the soot with nitric acid forms $-\mathrm{OH}$ and $\mathrm{CO}_{2} \mathrm{H}$ groups on the CQDs that makes them negatively charged and hydrophilic. The in vitro experiments showed that the CQDs with no further functionalization can enter into the cells and emit fluorescence. By UV excitation the cells emitted blue-green, and by blue excitation emitted yellow color emission, while the sample without CQDs was colorless under the aforementioned excitations. ${ }^{\mathbf{1 2 3}}$

The synthesized CQDs in most of the cases are filtered and collected from larger particles by dialysis tube or other filtration techniques. The dialysis tube pore size or its molecular weight cut-off (MWCO) has a significant influence on the emission properties of the resultant solution. It has been reported ${ }^{\mathbf{1 2 4}}$ that the excitation wavelength for the oxidized CQDs that were separated by dialysis membranes with MWCO of 3500 to $14000 \mathrm{Da}$, varies from 360 to $460 \mathrm{~nm}$. The CQDs that were collected by the aid of the membranes with MWCO > 3500 Da (up to $7000 \mathrm{Da}$ ) had similar emission intensities, while them that were collected by the membranes with MWCO of 7000$14000 \mathrm{Da}$, had decreased emission intensity. The CQDs that were collected using a membrane with MWCO of less than 3500 Da had low QY, since the particles came through the dialysis tube, had salts residues with them. The CQDs obtained from dialysis of MWCO of 3500-7000 had better optical properties. $^{\mathbf{1 2 4}}$ 

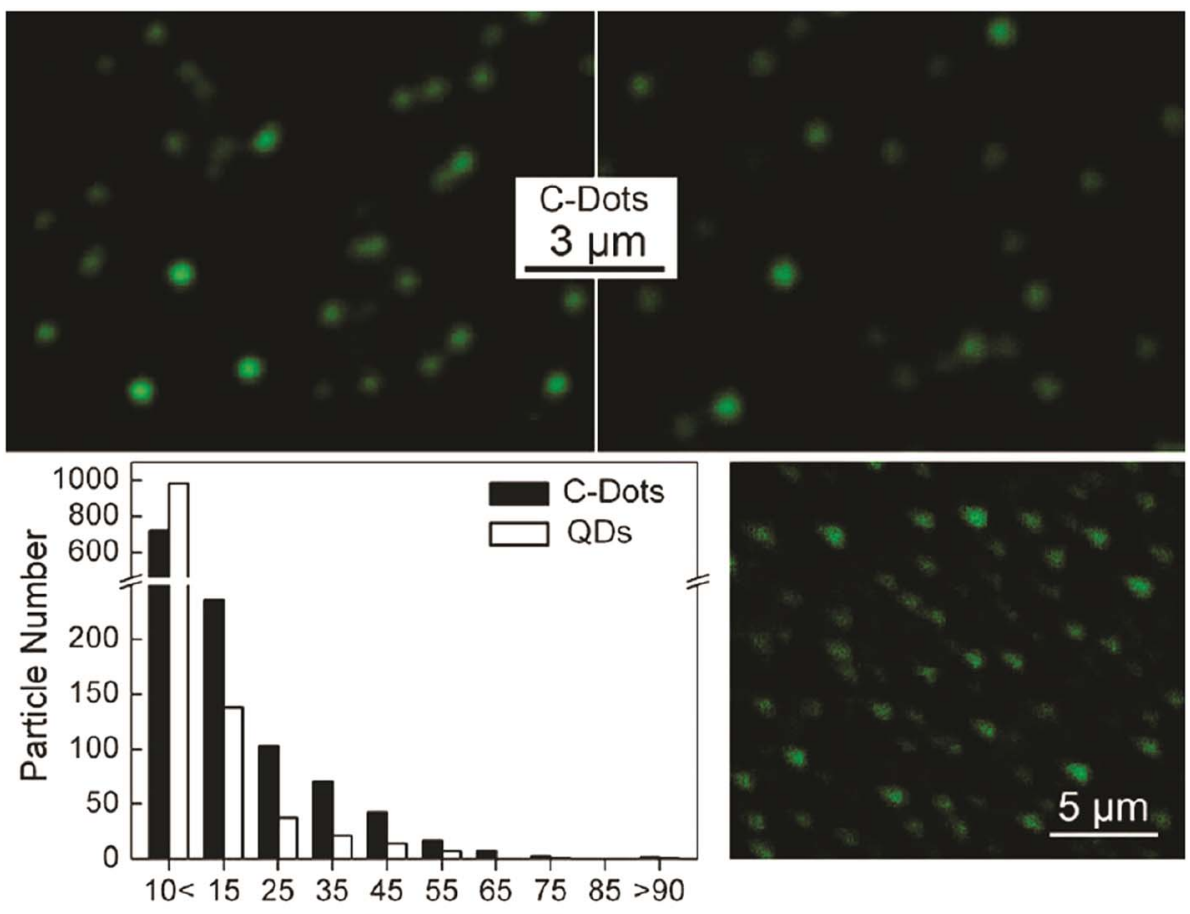

Normalized Intensity

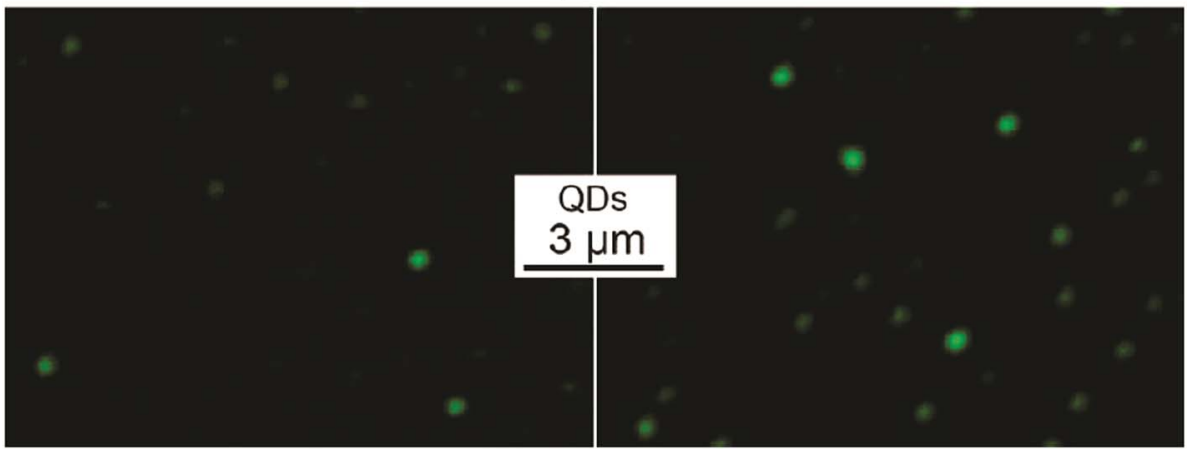

Fig. 3 Fluorescence images of CQDs (top) and CdSe/ZnS commercial QDs (bottom) in the infinite dilution conditions derived from numerous QDs in several images and their relative fluorescence intensity with excitation at $458 \mathrm{~nm}$ (middle left); the two-photon fluorescence intensity image by excitation at $880 \mathrm{~nm}$ (middle right), reprinted with permission from ref. 114, copyright 2009 American Chemical Society.

The oxidation has an intense effect on the PL characteristics of the CQDs. For example, in chemically derived GO thin films that formation of isolated $\mathrm{sp}^{2}$ clusters in the $\mathrm{sp}^{3}$ matrix of carbon-oxygen atoms results in localization of electron-hole pairs and subsequent radiative recombination, it was found that the PL intensity is influenced by reduction treatment. The reduction treatment alters the small $\mathrm{sp}^{2}$ clusters and hence, changes the PL intensity. Therefore, the PL in graphene can be tuned through the engineering of the $\mathrm{sp}^{2}$ domains by controlled oxidation. ${ }^{125}$ Similar results were observed by $\mathrm{Hu}$ et al. ${ }^{126}$ that reported the introduction of oxidized branchedpolyethyleneimine result in a hard band gap by shaping the oxygen-carbon matrix of the CQDs. The CQDs that were synthesized through oxidation and hydrothermal treatment of BPEI, with the diameter of $3-4 \mathrm{~nm}$ could reach a QY of $54.3 \%$. In the in vitro experiments of incubation of CQDs with MCF-7 cells, by excitation at $405 \mathrm{~nm}$, the luminescence was observed from inner the cells. The CQDs distributed uniformly in the cytoplasm. The PL from the CQDs in the cells did not decrease during 30 min excitation that is evidence of low photobleaching and superior photostability of the synthesized CQDs. ${ }^{126}$

Fluorescence emission of carbon nanoparticles and application for bioimaging is not confined to the nanoparticles with the sizes below $10 \mathrm{~nm}$. Larger carbon nanoparticles can also emit fluorescence. It has been reported ${ }^{127}$ that the carbon nanospheres with a mean particle size of $70 \mathrm{~nm}$ emit fluorescence and can be used for bioimaging. The fluorescent carbon nanospheres were synthesized via hydrothermal treatment of cocoon silk. The hydrothermal treatment causes the silk fibers to cut into shorter ones that by continues heating under hydrothermal conditions $\left(200^{\circ} \mathrm{C}\right)$ convert to irregular polymeric fragments and finally to larger and denser carbon nanoparticles. On the other hand, a bottom-up and top-down route, together are responsible for the formation of the carbon nanoparticles. After incubation of human cervical cancer (HeLa) cells with carbon nanoparticles, intracellular 
fluorescence was observed. The confocal laser scanning microscopy images showed that the fluorescence can be seen from perinuclear regions of the cytosol that is evidence of excellent cell permeability of the carbon nanoparticles into the cells. The carbon nanoparticles have been used for tissue imaging of human tumor tissues (MCF-7). The uniform distribution of fluorescence intensity in the tissue confirms the tissue permeability for carbon nanoparticles. The carbon nanoparticles have photostability with low bleaching and no blinking. ${ }^{127}$ Another example of the larger fluorescent nanoparticles that have been used for bioimaging is hollow carbon nanoparticles. The cross-linked hollow carbon nanoparticles with a shell thickness of $<5 \mathrm{~nm}$ and overall size of $105 \mathrm{~nm}$ were used for bioimaging. The carbon nanoparticles were synthesized by mixing of acetic acid (AC), diphosphorus pentoxide and water under self-generated heat. The bioimaging investigations with laser scanning confocal microscopy showed that the hollow carbon nanoparticles have excellent photostability and the intensity of the fluorescence did not change considerably during periods of time. In comparison with the organic dye (fluorescein isothiocyanate (FITC), Hoechst) and CdTe QDs, the cells labeled with hollow carbon nanoparticles were detectable even after $25 \mathrm{~min}$ which was not the case for the two other studied fluorescent agents. ${ }^{128}$

The CQDs have photostability that is beneficial in bioimaging applications. It has been reported that the PL intensity of the CQDs in the COS-7 cells after incubation for $24 \mathrm{~h}$ and subsequent penetration and labeling the cell membrane and the cytoplasm, had no obvious reduction upon continues excitation for $10 \mathrm{~min}$. This was attributed to the minor photobleaching and high photostability of the synthesized CQDs. ${ }^{129}$

The CQDs have low cytotoxicity even if are synthesized from toxic ingredients. For example, the CQDs that were synthesized from halophenols (a group of industrial pollutants) residual were used as fluorescent labeling agents. The CQDs were incubated with HeLa cells and the cells were excited with a $405 \mathrm{~nm}$ laser. The cell membrane and cell cytoplasm around the nucleus emitted fluorescence. The fluorescence intensity inside the cell nucleus was weak. Incubation of the CQDs with cells resulted in no morphological damage to the cells that show the CQDs have low cytotoxicity. ${ }^{\mathbf{1 3 0}}$

Plants, fruits and food products such as orange juice, ${ }^{\mathbf{1 3 1}}$ apple juice, ${ }^{\mathbf{1 3 2 , 1 3 3}}$ cabbage, ${ }^{\mathbf{1 3 4}}$ Carica papaya juice, ${ }^{\mathbf{1 3 5}}$ banana juice, ${ }^{136}$ sucrose,${ }^{37}$ unripe peach, ${ }^{137}$ waste frying oil, ${ }^{138}$ gelatin, ${ }^{62}$ milk, ${ }^{139}$ soy milk, ${ }^{140}$ honey, ${ }^{141}$ egg $^{142}$, flour, ${ }^{40}$ coffee grounds, ${ }^{143}$ sugarcane bagasse pulp ${ }^{\mathbf{1 4 4}}$ and etc. have been also used as the carbon source in the synthesis of CQDs. Using natural sources and food products may result in lower toxicity of the synthesized CQDs for biomedical applications. Mehta et al. ${ }^{145}$ used Saccharum officinarum juice as the carbon source for production of CQDs. The synthesized CQDs were used for cell imaging in $E$. coli (bacteria) and $S$. cerevisiae (yeast). The laser confocal fluorescence microscopic studies showed that different colors of red, green and blue can be obtained from the labeled bacteria and yeast cells by using $40 \mu \mathrm{g} \mathrm{mL}^{-1}$ of the CQDs probes and applying different excitations wavelengths. The $E$. coli confocal images showed that the CQDs are well dispersed in the membrane and cytoplasm. The CQDs were taken by the cells via endocytosis mechanism. The CQDs were located in the yeast nucleus keeping alive $>90 \%$ of the yeast cells. ${ }^{145}$ Orange juice was also used in synthesizing CQDs for bioimaging applications. The CQDs with a QY of $26 \%$ were synthesized through a hydrothermal route. The CQDs were used for imaging in human osteosarcoma (MG-63) cells. The CQDs were agglomerated in the cells while missed reaching to the nuclei. The PL of the CQDs shows excitation-dependent emission. The PL intensity for the synthesized CQDs in bioimaging does not show reduction via prolonged excitation time. ${ }^{\mathbf{1 4 6}}$

The N-doping of CQDs has been used as a tool for enhancing the PL properties. N-doping has broadened the CQDs applications. The higher PL efficiency results in the images with improved resolution. The CQDs that were N-doped through different nitrogen precursors of diamines showed increased QY and were used as labeling agents for bioimaging. The $\mathrm{N}$-doping resulted in a QY as high as $36.3 \%$. Fluorescence images (with an excitation wavelength of $355 \mathrm{~nm}$ ) of human HeLa cells showed the CQDs that were incubated with the cells could label the cytoplasm whereas the area that was labeled with $\mathrm{N}$-doped CQDs was brighter compared to the CQDs without N-doping. On the other hand, labeling with $\mathrm{N}$-doped CQDs can be done at lower doses. Furthermore, prolonged UV exposure caused the fluorescence of CQDs to be bleached after $15 \mathrm{~min}$ while the fluorescence of the N-doped CQDs was still bright at the same time. It implies that the N-doped CQDs have better resistance to photobleaching. ${ }^{\mathbf{1 4 7}}$

Doped nitrogen and low oxidation level can improve the fluorescence properties in the CQDs. It has been shown that the emission intensity is affected by the doped nitrogen content. The N-doped CQDs were prepared through carbonization of some precursors such as 3-(3,4-dihydroxyphenyl)-L-alanine ( $\mathrm{L}$ DOPA), L-histidine, and L-arginine. The CQDs consisted of a carbon core and oxygen-containing groups of the amorphous shell. It was observed that the nitrogen-carbon bonding resulted in PL improvement. The emission wavelength was dependent on the CQDs size while the emission intensity was dependent on the nitrogen content. The CQDs were used for imaging of HeLa and HepG2 cells. The cells that were incubated with N-doped CQDs showed images with better resolution in confocal fluorescence imaging compared to other CQDs. The Ndoped CQDs were accumulated mostly in the cytoplasm. ${ }^{\mathbf{1 2 4}}$ In another experiment, the $\mathrm{N}$-doped carbon nanospheres synthesized from hydrothermal treatment of cocoon silk in water were used for the imaging of HeLa cells that were incubated with $\mathrm{N}$ doped CQDs. The applied CQDs in bioimaging have photostability with low bleaching and have no blinking. ${ }^{\mathbf{1 2 7}}$

The fluorescence of the CQDs can be applicable in the NIR region (wavelength of 700-900 $\mathrm{nm}$ ) imaging. The NIR region imaging has the benefit of an ideal tissue transparency window for bioimaging in vivo. In spite of the fact that fluorescence emission of CQDs at longer wavelengths is weaker, the signalto-noise ratio would be improved due to the decrease in the tissue autofluorescence by excitation at red and NIR region of the spectrum. Better photon tissue penetration in bioimaging at longer wavelengths is also another reason for preference of 
imaging at this range. ${ }^{148}$ The CQDs that were prepared from the precursor of polythiophene phenylpropionic acid (PPA) exhibited red emission with absorption in the range of 400-800 nm (visible-NIR). Using laser irradiation, the synthesized CQDs also have the photo-thermal efficiency of $38.5 \%$. The synthesized CQDs show fluorescence emission via visible light excitation (350-600 nm) ${ }^{149}$ Furthermore, the CQDs synthesized from the oxidation of different carbon sources (graphite and carbon nanotubes) with mixed acids showed fluorescence by excitation in the range of visible to NIR.

Down-conversion fluorescence occurs in some fluorescence materials such as semiconductor QDs and organic dyes. These materials absorb a higher energy photon and then emit a lower energy fluorescence photon. Absorption of higher energy photon has the drawbacks of auto-fluorescence of biological samples, low penetration depth and in some cases intensive damage to the organisms by high energy photons. ${ }^{150}$

The photon upconversion occurs when two or more lowenergy photons are sequentially absorbed to emit high-energy luminescence. The upconversion is optically nonlinear. The upconversion applications range from 3D flat panel displays and solid-state lasers to bioimaging and bio-labeling. Lanthanide-doped upconversion nanoparticles (UCNPs) have several advantages over conventional biological labels such as large anti-Stokes shifts, high signal-to-noise ratio, narrow emission bandwidths, resistance to photo-bleaching, and excellent tissue penetration. ${ }^{151}$ CQDs show photon upconversion that facilitates their application in bioimaging in the NIR region of the spectrum. The upconverted NIR multiphoton bioimaging has the advantage of preventing autofluorescence from tissues. The electrochemically synthesized CQDs with a mean diameter of $6 \mathrm{~nm}$ that were excited with the wavelengths in the range of 600-800 $\mathrm{nm}$, showed emission in the range of 405 to $565 \mathrm{~nm}$ that this phenomenon is due to multi-photon absorption. ${ }^{150}$ The CQDs obtained from heating of the ascorbic acid aqueous solution also showed upconversion optical properties. The excitation of the synthesized CQDs at the NIR region showed a fixed emission (at $540 \mathrm{~nm}$ ) that in contrast to other reports, did not change considerably by changing the excitation wavelength (Fig. 4). The nearly constant emission spectra for each excitation wavelength is due to the emission from the lowest single state. ${ }^{152}$ The excitation of N-doped CQDs with wavelengths in the range of 700-800 $\mathrm{nm}$ resulted in the emission profile with bandwidths and peak wavelengths almost similar to normal PL (excitation at $380 \mathrm{~nm}$ ) but with a twofold excitation wavelength. ${ }^{124}$

GQDs can also exhibit upconversion. The GQDs that were excited at $980 \mathrm{~nm}$, had upconverted PL emission at $525 \mathrm{~nm}$. When the electrons of the $\pi$ orbital are excited by a bunch of low energy photons, the $\pi$ electrons would experience a transition to higher energy level such as lowest unoccupied molecular orbital (LUMO) and then, the electrons will return back to lower energy state. Therefore, by transition back of the electrons to the $\sigma$ orbital, upconverted PL would emit. The $\sigma$ orbital electrons can also be transitioned. However, the $\sigma$ orbital electrons can only emit normal PL. ${ }^{153}$
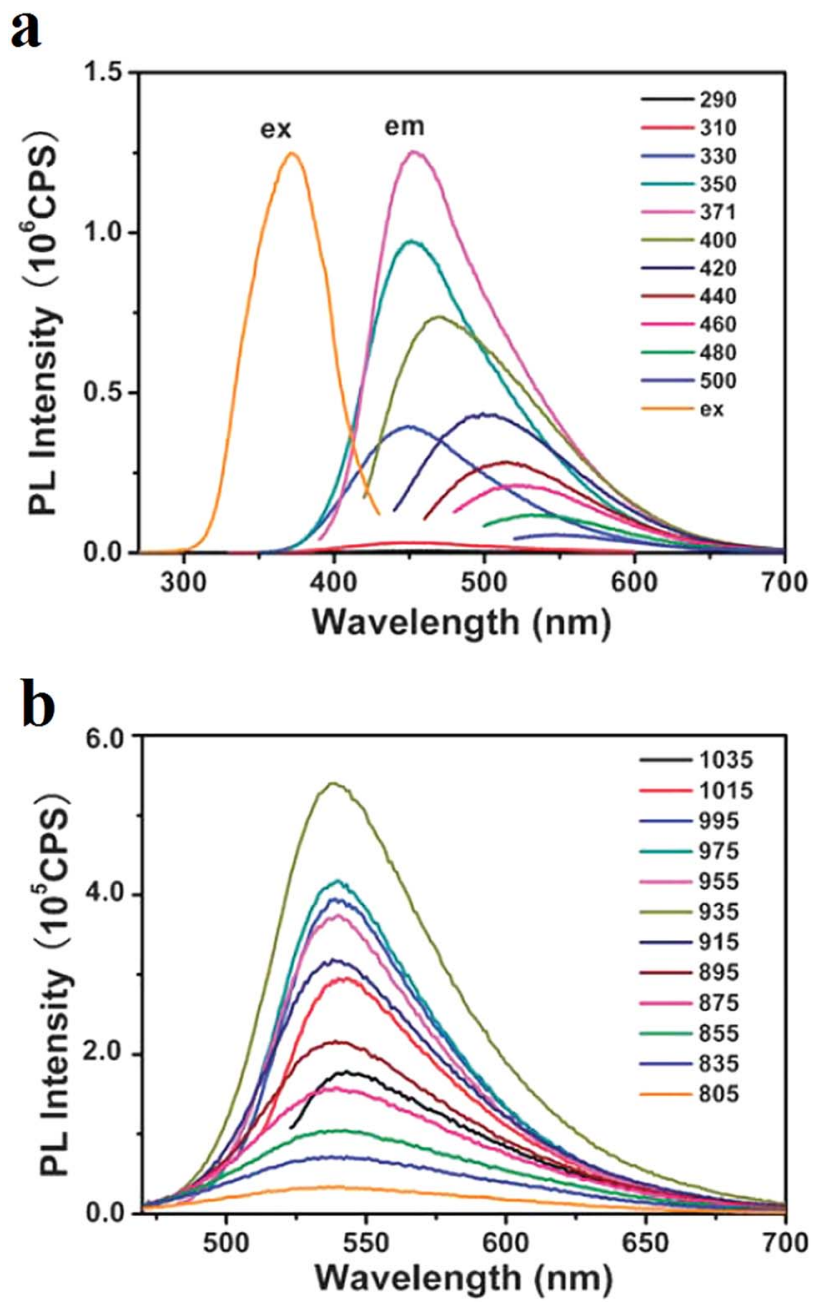

Fig. 4 Excitation and emission spectra of the CQDs that were synthesized via heating of the ascorbic acid aqueous solution; (a) emission spectra at excitations ranging from $290 \mathrm{~nm}$ to $500 \mathrm{~nm}$, (b) upconversion seen in the emission spectra of the CQDs, the PL emission spectra for excitations from $805 \mathrm{~nm}$ to $1035 \mathrm{~nm}$, reprinted with permission from ref. 152, copyright 2012 Royal Society of Chemistry.

4.1.2. Multicolor photoluminescence. The available CQDs are mostly fluorescent in the green or shorter wavelengths regions of the light spectrum that might be less suitable for bioimaging applications. ${ }^{3}$ However, some CQDs similar to the semiconductor QDs, molecular nanomaterials, rare-earth based nanoparticles, PDs, and organic fluorescent dyes can emit red, green, and blue (RGB, the main primary colors) PL. For example, the CQDs that are prepared by heating of three isomers of phenylenediamine, i.e. $o$-phenylenediamine (oPD), $m$-phenylenediamine (mPD), and $p$-phenylenediamine (pPD) in ethanol solution in an autoclave at $180{ }^{\circ} \mathrm{C}$ that can be dispersed within different common solvents showed multi-color fluorescence emission. For example, if dispersed in ethanol, they form colorless, yellow and pink solutions by using the CQDs derived from $\mathrm{mPD}$, oPD, and $\mathrm{pPD}$, respectively. Interestingly, each solution shows an emission for one of the primary colors (Fig. 5). The prepared composite films of PVA and the 


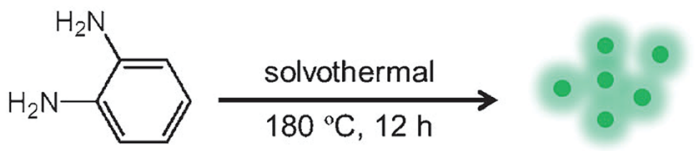

o-CDs

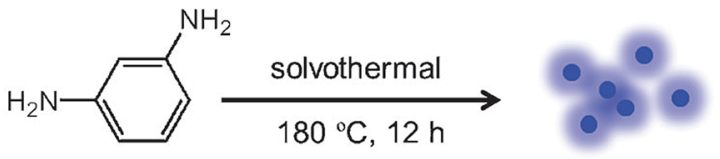

$m$-CDs<smiles>Nc1ccc(N)cc1</smiles>

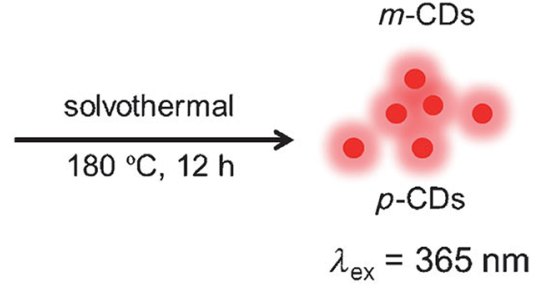

b

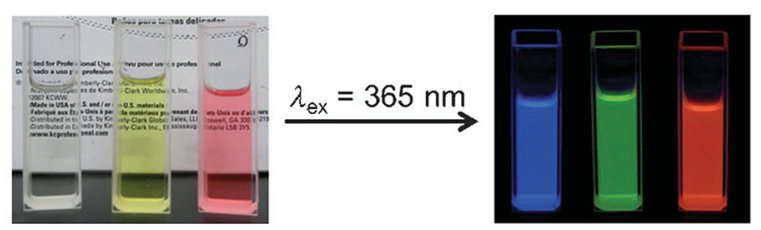

c

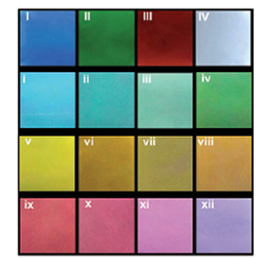

d

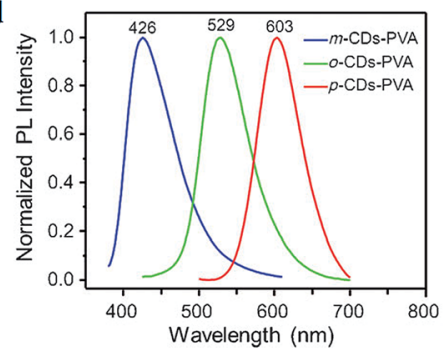

e
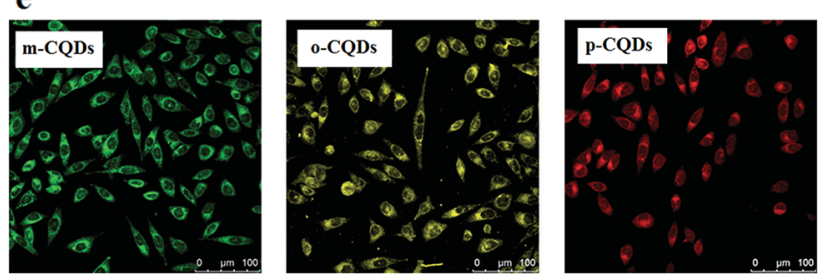

Fig. 5 (a) Synthesis method of CQDs from phenylenediamine isomers, OPD, $\mathrm{mPD}$ and $\mathrm{pPD}$ that results in $o-C Q D s, m-C Q D s$, and $p$-CQDs, respectively. (b) Photographs of the ethanol solutions of $o-C Q D s, m-$ CQDs, and $p$-CQDs in daylight (left) and under UV excitation with the wavelength of $\lambda=365 \mathrm{~nm}$ (right), (c) the $m$-CQDs, o-CQDs, $p$-CQDs mixtures in PVA films under excitation with a wavelength of $\lambda=$ $365 \mathrm{~nm}$ : (I) $\mathrm{m}$-CQDs; (II) o-CQDs; (III) $p$-CQDs; (IV) o-CQDs $/ m$ CQDs/p-CQDs $=2: 4: 1(\mathrm{w} / \mathrm{w} / \mathrm{w}) ;(\mathrm{i}-\mathrm{iv}) o-C Q D s / m-C Q D s=1: 8$, $1: 4,1: 2$, and $1: 1$; (v-viii) o-CQDs/p-CQDs = 4:1, $2: 1,1: 1$, and $1: 2$; (ix-xii) $m$-CQDs/p-CQDs $=1: 4,1: 2,1: 1$, and $4: 1$ (all ratios are $\mathrm{w} / \mathrm{w}),(\mathrm{d})$ the normalized PL spectra of the PVA films with $m$-CQDs, $0^{-}$ CQDs, and $p$-CQDs (UV excitation of $\lambda=365 \mathrm{~nm}$ ), (e) confocal fluorescence microscopy of $m$-CQDs, $0-C Q D s$ and $p$-CQDs of MCF-7 cells at a constant laser excitation wavelength of $405 \mathrm{~nm}$, reprinted with permission from ref. 154, copyright 2015 John Wiley and Sons.

synthesized CQDs with various ratios of $m$-CQDs, $o$-CQDs, and $p$-CQDs derived from the $\mathrm{MPD}$, oPD, and $\mathrm{pPD}$, respectively, result in making full-color PVA films. The films show full-color emission while they are excited at a single wavelength of $\lambda=$ $365 \mathrm{~nm}$ (Fig. 5c). It was observed that the significant interaction between different CQDs in the mixture of the PVA films cannot occur and hence, using Glassman color mixing law, full-color emissions can be made by using CQDs with three primary colors. The CQDs were incubated with MCF-7 cells for 4 hours and under excitation of a $405 \mathrm{~nm}$ laser of a confocal microscope, different colors were emitted from different CQDs. The emissions are mostly from cytoplasmic regions. Therefore, the three different CQDs can pass the cells membranes (Fig. 5e). ${ }^{154}$

There have been some efforts to manipulate the CQDs in order to emit at longer wavelengths like red and NIR. ${ }^{3}$ The CQDs with blue, green, yellow and red emissions were synthesized through different controlled carbohydrate carbonization methods. The synthesized CQDs were applied in functionalized nanoprobes through bioconjugation chemistries. The synthesized CQDs can emit different color lights similar to classical semiconductor QDs, while they are much less toxic (Fig. 6). ${ }^{155}$

The CQDs that have been functionalized with polyethyleneimine (PEI) showed multicolor emission under different excitation wavelengths. By changing the excitation wavelength from $340 \mathrm{~nm}$ to $500 \mathrm{~nm}$, the functionalized CQDs emission shifted from $450 \mathrm{~nm}$ (blue color) to $550 \mathrm{~nm}$ (green) while the PL intensity decreased. The dependence of the CQDs PL emission on the excitation wavelength has been attributed to the distribution of different emissive energy traps on the surface of the CQDs. ${ }^{156}$

4.1.3. In vivo imaging. The CQDs have been used for in vivo imaging as well. ${ }^{61,157-161}$ Due to minor toxicity, the CQDs are superior candidates for in vivo imaging instead of other heavy metal containing QDs. The CQDs that are derived from different carbon sources and are functionalized with appropriate functional groups have the potential to emit under excitation in UV or NIR regions of the light spectrum.

For in vivo optical imaging it is preferred to use longer wavelengths since the photon-tissue penetration increases and the background autofluorescence decreases. The CQDs fluorescence emission might be weaker at longer wavelengths but the imaging at these wavelengths can have the benefit of reduced tissue autofluorescence background which in turn, results in improved signal-to-noise ratio. For example, the CQDs that were produced through oxidization of carbon nanotubes with the size of 3-4 nm were used for in vivo experiments on the mouse. The CQDs were used to administer a nude mouse at three different locations. Through excitation with blue, green, yellow, orange, red, deep red and NIR light and differentiating the green background autofluorescence, the red spots were seen in the fluorescence images (Fig. 7). The images show that compared to those taken under blue and green excitations, the images that are obtained by excitation at $595 \mathrm{~nm}$ and beyond, have improved signal-to-background separation. ${ }^{148}$

The CQDs have some advantages in comparison to other commercially available QDs for bioimaging due to their absorptivity. The higher absorptivity of CQDs can compensate for their lower fluorescence yield compared to the heavy metal containing QDs. The PEG surface passivated CQDs were compared for optical imaging in reference to CdSe/ZnS QDs. 

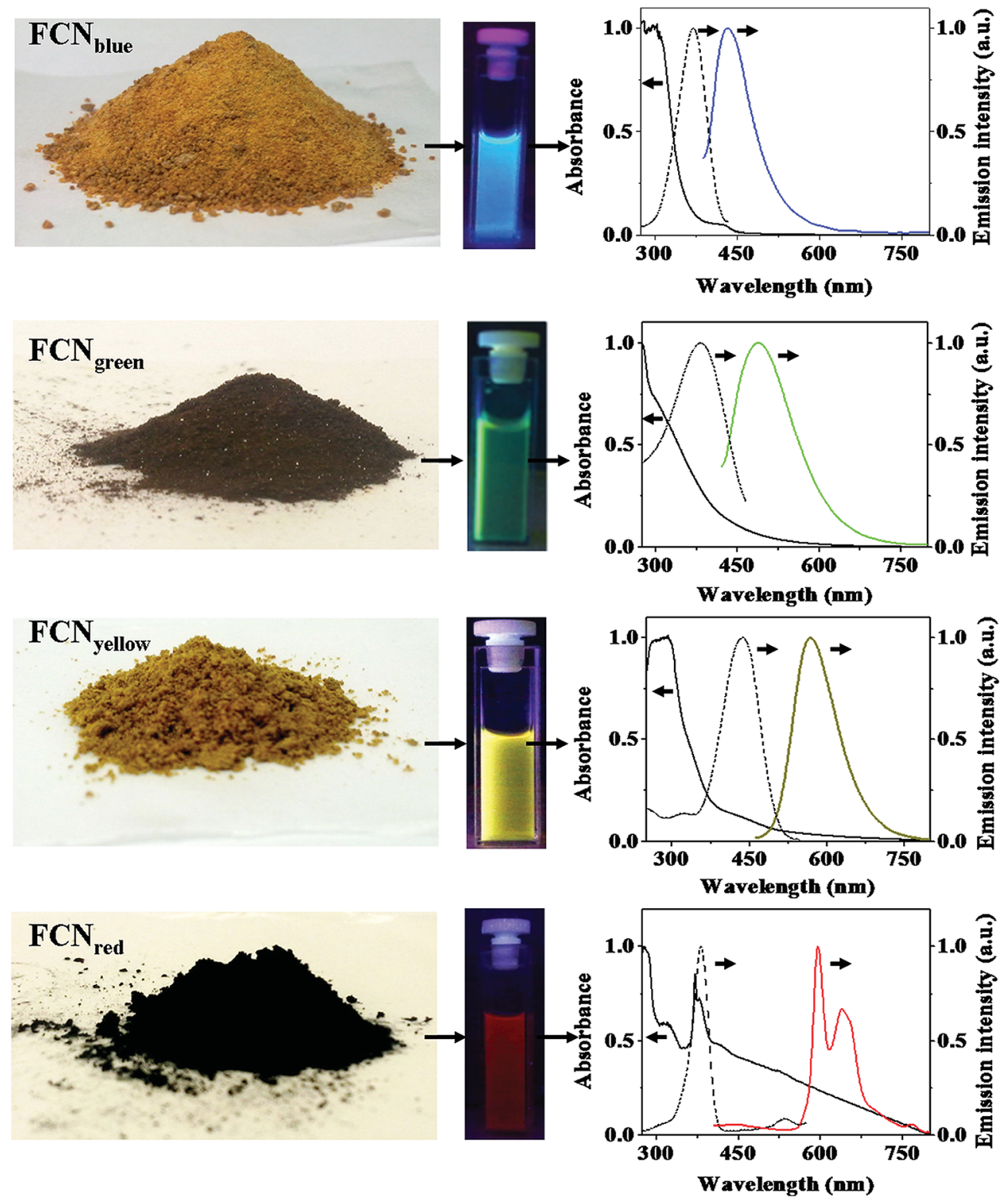

Fig. 6 Different CQDs synthesized through different controlled carbohydrate carbonization methods, the solution containing CQDs and regarding absorption (solid line), excitation (dashed line) and emission (colored line) spectra; the emission spectra are recorded by excitation at $370 \mathrm{~nm}$ for blue CQDs, at $400 \mathrm{~nm}$ for green CQDs, at $425 \mathrm{~nm}$ for yellow CQDs, and at $385 \mathrm{~nm}$ for red CQDs, reprinted with permission from ref. 155, copyright 2013 Springer Nature.

The PEG passivated CQDs are an appropriate candidate for in vivo optical imaging since are nontoxic and biocompatible. The surface passivated CQDs have better absorptivity and potential isolating of the dots for improved bioimaging. The smaller size of the CQDs makes them appropriate for tracing small molecules and proteins and capable of tracing fine biological structures. ${ }^{114}$ Surface passivation has an obvious effect on the fluorescence properties of the CQDs used in vitro and in vivo imaging. For example, it is reported that NIR emission of the CQDs that were passivated with a hyperbranched polymer increased compared to the CQDs that were coated with a linear polymer (PEG). The in vivo experiments on a mouse model resulted in contrast enhancement of the fluorescence emission in auxiliary lymph node. ${ }^{162}$

The CQDs without any functionalization can also cross the blood-brain barrier (BBB) and enter the brain. In an attempt of bioimaging using CQDs, the fluorescence emission showed that the CQDs enter different organs such as lung, liver, spleen, heart, and kidney. The excitation can be at longer wavelengths to result in a bright blue emission (Fig. 8A). Compared to other organs, the spleen had enhanced fluorescence emission with high intensity. However, some of the CQDs were observed in the brain. Fig. 8B confirms the CQDs presence inside and around a single neuron under CLSM. ${ }^{163}$ 


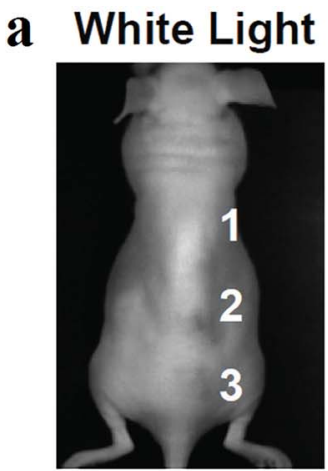

$605 \mathrm{~nm}$

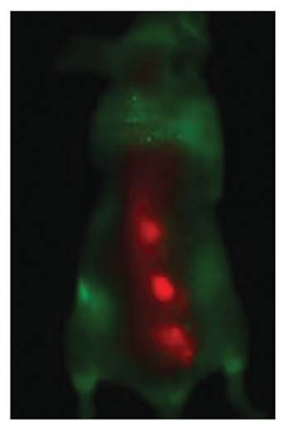

$635 \mathrm{~nm}$

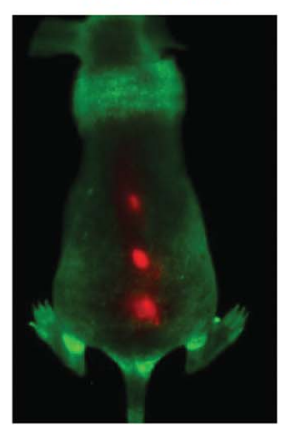

$523 \mathrm{~nm}$

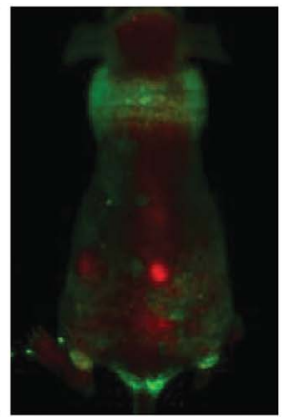

$661 \mathrm{~nm}$

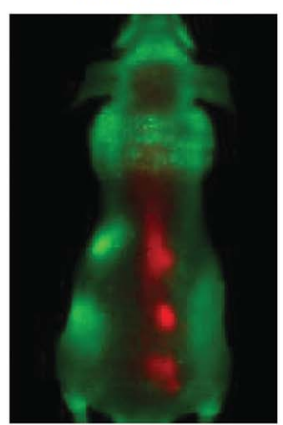

$595 \mathrm{~nm}$

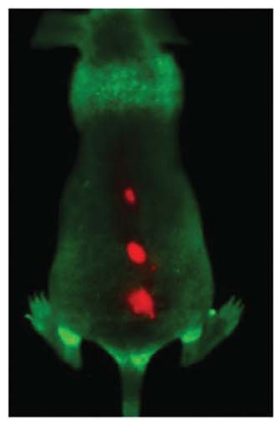

704 nm

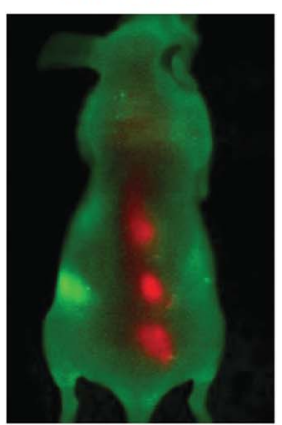

b

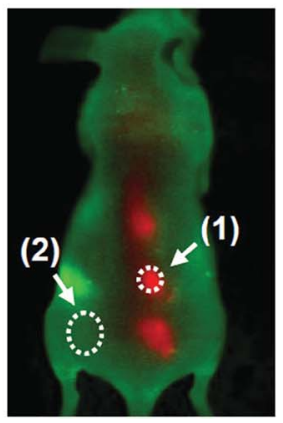

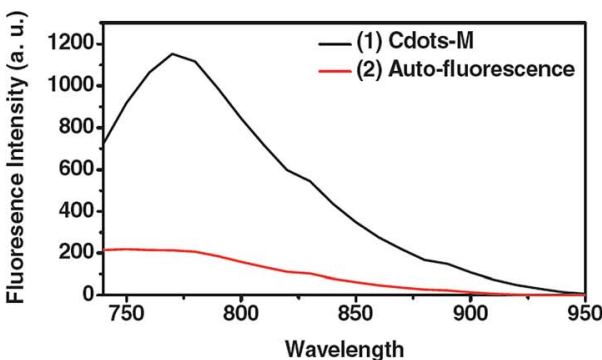

Fig. 7 (a) The in vivo fluorescence images of CQDs that are injected into a nude mouse; the excitation wavelengths are indicated above each image. The fluorescence signal and tissue autofluorescence can be seen in red and green emissions, respectively. (b) Signal-to-background separation of the image taken under $704 \mathrm{~nm}$ excitation, reprinted with permission from ref. 148, copyright 2011 John Wiley and Sons.

The CQDs may be selectively uptake by macrophages in the spleen. The selective uptake of CQDs in spleen (and not in the liver and kidney) might be due to blood filtration by the spleen. The CQDs size is very small and they might phagocytize by the splenic reticuloendothelial cells and therefore, minor amounts of these small particles would remain for other organs such as liver tissue. ${ }^{\mathbf{1 6 4}}$

The CQDs could be doped with inorganic salts (e.g. ZnS) to improve the fluorescence properties for in vivo bioimaging. Injection of a solution of the CQDs that were doped with $\mathrm{ZnS}$ (C-ZnS) into the mice, compared to the un-doped CQDs showed that during the fluorescence imaging, the former can result in a brighter emission (Fig. 9). The C-ZnS with a green fluorescence upon intradermal injection into the front extremity migrates along the arm. The migration of the $\mathrm{C}-\mathrm{ZnS}$ is slow in comparison to conventional semiconductor QDs. The reason is smaller sizes of the $\mathrm{C}-\mathrm{ZnS}$ and/or the PEG functionalization that results in reducing the dots interaction with lymph cells. ${ }^{\mathbf{1 6 5}}$
4.1.4. Photoacoustic imaging. The CQDs can act as a NIR light-triggered photoacoustic (PA) imaging in vivo. The PA imaging uses the integration of optical excitation with ultrasonic detection that leads to imaging beyond optical imaging diffusion limits. Therefore, integration of fluorescence and PA imaging within a single probe can result in a deeper tissue penetration with a high imaging sensitivity. ${ }^{149}$ The optical scattering can be overcome ultrasonically and centimeters penetration depth with an improved spatial resolution can be obtained. ${ }^{166}$ On the other hand, the PA can result in highresolution structural images of the tumor and fluorescence imaging can have the benefit of tumor accurate detection with molecular probing. For example, the CQDs that were injected intravenously into the tumor-bearing nude mice were accumulated in the tumor through enhanced permeability and retention (EPR) effect. The fluorescence that was observed in the tumor area was pronounced over other normal tissues 

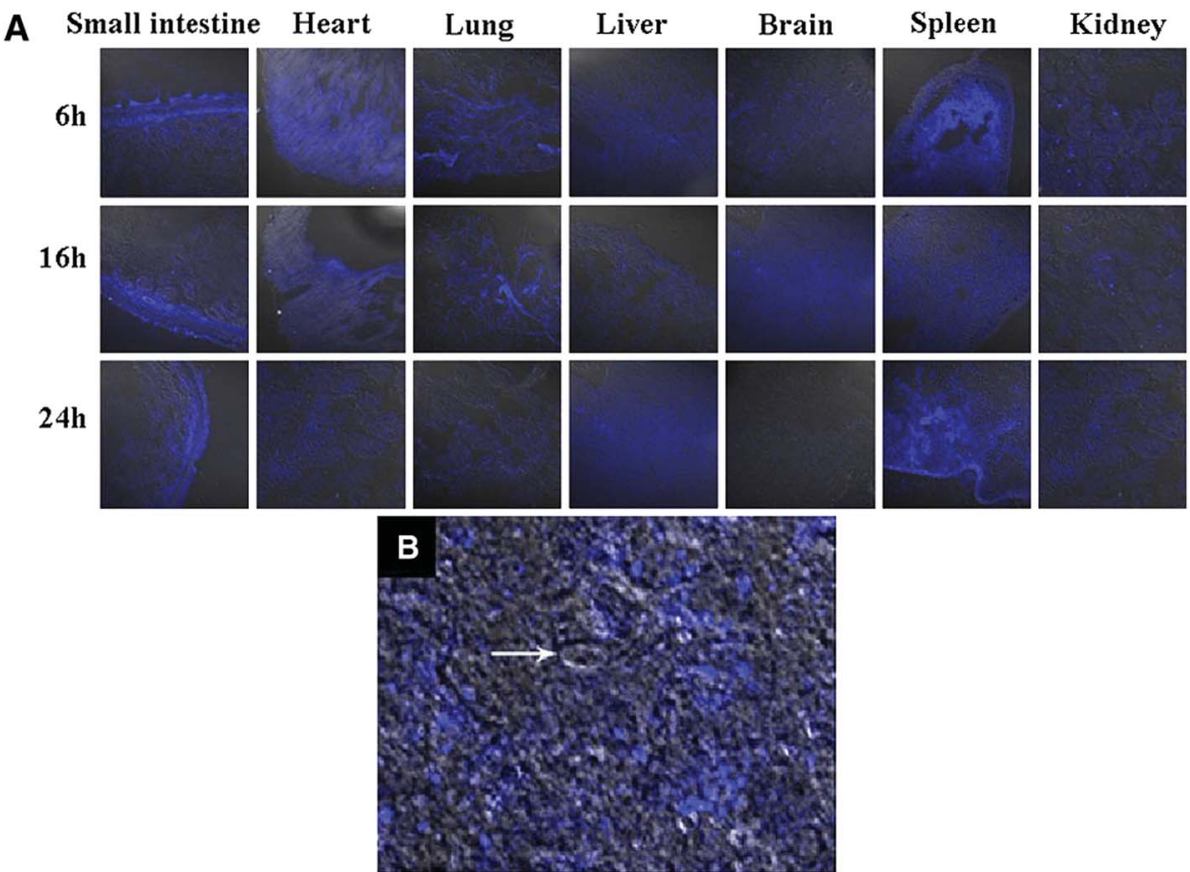

Fig. 8 (A) The in vivo images of the different organs of Kunming mice which are injected with CQDs at different post-injection periods (absorption wavelength of $405 \mathrm{~nm}$ and an emission wavelength of $500 \mathrm{~nm}$ ), (B) the CQDs inside and around single neuron under CLSM; the arrow shows nucleus, reprinted with permission from ref. 163, copyright 2012 Springer Nature.

(Fig. 10a). The major organs of mice and the tumors were excised and imaged at $24 \mathrm{~h}$ post-injection. While the fluorescence emission from the spleen and heart were weak, the excised tumor tissue showed the high intensity of fluorescence. The CQDs were accumulated in the liver, lung, and kidney tissues (Fig. 10b). The fluorescence intensity of the CQDs in the tumor area increased rapidly within $5 \mathrm{~h}$ post-injection (Fig. 10c). The in vivo PA imaging showed that the CQDs accumulate in the tumors with constant PA signals during long circulation time (Fig. 10d). The signal intensity increases with time until $8 \mathrm{~h}$ after injection. Therefore, $8 \mathrm{~h}$ after injection would be the optimum time for photothermal ablation of the tumor (Fig. 10e). ${ }^{149}$

\subsection{Drug delivery}

Drug delivery systems (DDSs) require designing systems which are capable of transporting the drug to a specific target in the body and proper interaction of the drug with the target. Nanostructured materials in conjugate with the drug(s) can improve the DDSs with respect to the drugs absorption, distribution and
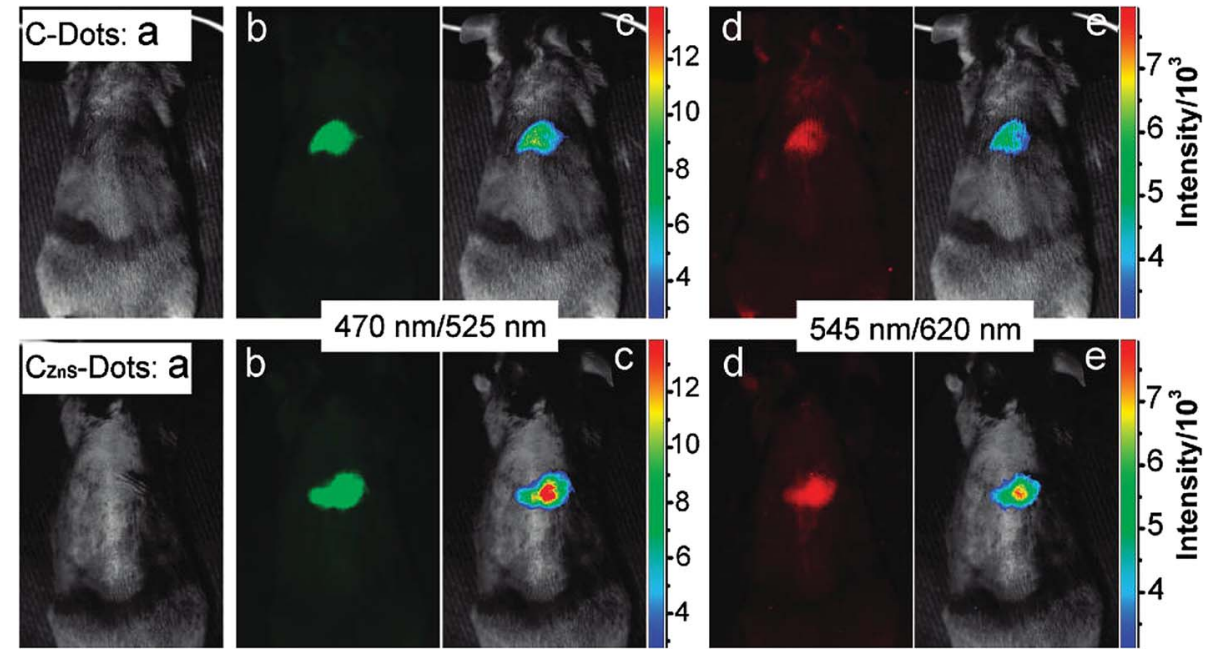

Fig. 9 Comparison of CQDs (top) and C-ZnS dots (bottom) after subcutaneous injection (a) bright field, (b and d) as-detected fluorescence, (c and e) color-coded images (the excitation/emission wavelength are indicated on the images), reprinted with permission from ref. 165, copyright 2009 American Chemical Society. 

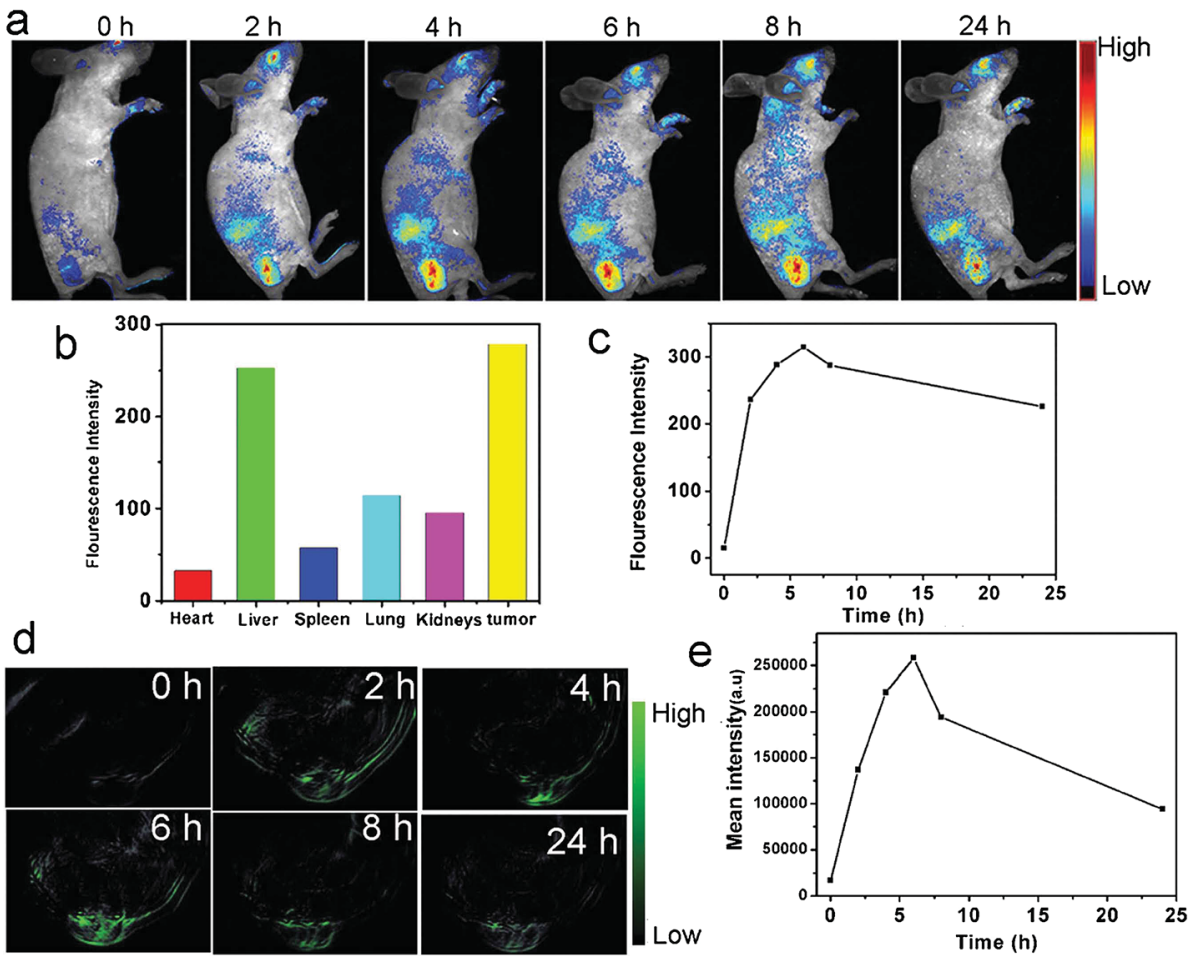

Fig. 10 The in vivo fluorescence imaging and PA imaging of the mice injected with CQDs, (a) the real-time red fluorescence images of the mice after i.v. injection of CQDs at different post-injection times, (b) the fluorescence intensities of different areas $24 \mathrm{~h}$ after the CQDs injection, (c) the variation of average fluorescence intensity emitted from tumor during time, (d) the PA imaging of the tumor (in mice) after 0, 2, 4, 6, 8 and 24 h post-injection, (e) mean intensity of the tumor as a region of interest (ROI) up to $24 \mathrm{~h}$ post-injection, reprinted with permission from ref. 149 , copyright 2015 John Wiley and Sons.

elimination. ${ }^{\mathbf{1 6 7}}$ Application of nanotechnology in DDSs may result in drug targeting to a specific cell or tissue, enhanced delivery of drugs with low water solubility, delivery of two or more drugs or therapeutic modality for different therapy simultaneously, transferring of large macromolecule drugs, and monitoring of the drug site using imaging agents on the drug carrier. $^{168}$

Recently, CQDs have received increasing attention for drug delivery due to their superior properties such as fluorescence emission, small size and resultant cell membrane permeability, low toxicity, chemical inertness, water solubility, easy synthesis, potential functionalization, and drug loading. Several researchers have applied CQDs in DDSs. ${ }^{\mathbf{1 6 9 - 1 7 2}}$ For example, Karthik et al. ${ }^{173}$ could bond 7-(3-bromopropoxy)-2quinolylmethyl chlorambucil (Qucbl) covalently to the nitrogen-containing CQDs. The FTIR spectroscopy confirmed that the drug has been loaded on the CQD surface. The in vitro experiments showed aggregation of the drug-loaded CQDs in the cytoplasm and nucleus. The drug-loaded CQDs had a controlled drug release to kill cancer cells by means of irradiation. In another set of experiments, dopamine hydrochloride (DA) was anchored to the CQDs to investigate the release profile of the drug-anchored CQDs in vitro. As compared to the control DA alone, the DA release in CQD-DA conjugate extended to $60 \mathrm{~h}$ while it was biocompatible for Neuro 2A cells. ${ }^{167}$

In drug delivery applications, hollow nanostructures have received attention for their efficacy in drug loading. ${ }^{.14-176}$ Hollow
CQDs with fluorescence emission properties have also been used for drug loading. In a set of experiments, doxorubicin (DOX) was loaded on the hollow CQDs. The in vitro experiments showed that the CQDs-DOX are rapidly taken up by the cells and show $\mathrm{pH}$-controlled release. The fluorescence microscopy confirmed that the CQDs-DOX were internalized into the A549 cells and localized mainly in the cytoplasm. However, the DOX was detected in the cell nucleus as well. In fact, the CQDs were able to release DOX into the nucleus. ${ }^{177}$

The CQDs used in DDSs can become magnetic to be used for magnetic resonance imaging (MRI) while having the benefit of drug delivery and fluorescence imaging. The MRI combined with fluorescence imaging would have the benefit of superior tissue penetration and spatial resolution of MRI and easy microscopic tissue examination of the fluorescent imaging. For example, magnetofluorescent CQDs have been synthesized through incorporating $\mathrm{Eu}^{3+}, \mathrm{Mn}^{2+}$, and $\mathrm{Gd}^{3+}$ ions into the dots. The transition metals dopants have unpaired electrons which result in a magnetic field under an applied magnetic field. The magnetofluorescent CQDs have been loaded with DOX in order to be used for drug delivery to the HeLa cells. ${ }^{\mathbf{8 1}}$

The CQDs have also been used for drug delivery in other researches, including passivated CQDs with polyaminecontaining organosilane molecules for delivery of $\mathrm{DOX},{ }^{\mathbf{1 0 5}}$ organophilic CQDs for anticancer drug curcumin delivery with high loading efficiency and rapid penetration into the HeLa cells, ${ }^{178}$ CQDs synthesized through hydrothermal treatment of 
pasteurized milk for delivery of lisinopril (Lis) to HeLa cells, ${ }^{\mathbf{1 7 9}}$ CQDs/calcium alginate hydrogel film for delivery of glycopeptides antibiotic vancomycin in the gastrointestinal tract, ${ }^{\mathbf{1 8 0}}$ CQDs/mesoporous silica nanocarriers for delivery of DOX, ${ }^{\mathbf{1 8 1}}$ functionalized and surface passivated CQDs for delivery of DOX to the MCF-7 cells, ${ }^{182}$ and CQDs/hydroxyapatite hybrid nanorods for delivery of DOX to the HeLa cells and human prostatic cancer (PC-3) cells. ${ }^{183}$

The CQDs have been used in multifunctional platforms for drug delivery and magnetic delivery and/or MRI agents and/or simultaneous co-delivery of two or more drugs, including multi-functional DDS of CQDs and DOX and heparin as an auxiliary medicine for delivery to the A549 cells, ${ }^{184} \mathrm{Fe}_{3} \mathrm{O}_{4} @$ @QDs coated single-walled carbon nanotubes loaded with DOX and conjugated with an sgc8c aptamer as a platform for combining cancer photothermal therapy, chemotherapy, and photodynamic therapy, ${ }^{\mathbf{1 8 5}}$ and magnetic gadolinium oxide-iron oxide core, mesoporous silica shell gated with boronic acid functionalized CQDs for drug delivery of anticancer drug 5-fluorouracil to HePG2 cancer cells which the platform acts as MRI agent and optical imaging agent as well as stimuli-responsive DDS. ${ }^{186}$

\subsection{Crossing blood-brain barrier}

An important and major parameter for using any kind of nanostructures in the living biomedical applications is the size of the nanoparticles. The smallest human capillaries are less than $4 \mu \mathrm{m}$. Therefore, it is essential to keep the size of the nanoparticles below this size for use in a living body. Smaller sizes of the nanoparticles would prevent blood vessels blockage and their elimination by the reticuloendothelial system. ${ }^{\mathbf{1 1 1}}$ Delivering of imaging probes to the brain tumors is a complex technique because of the Blood-Brain Barrier (BBB). Crossing the BBB depends on the size of the probes and their surface properties. ${ }^{18}$ Furthermore, delivering the drugs to the brain is difficult because of the BBB properties which prevent drugs such as foreign proteins, chemicals, and peptides to cross. ${ }^{\mathbf{1 6 7}}$ Nanoparticles and QDs can be used for drug delivery through crossing BBB. ${ }^{187-190}$ The CQDs may also be used for drug delivery across BBB as well. Li et al. reported that synthesized CQDs have been observed in all organs including the brain after $6 \mathrm{~h}$ postinjection. In fact, the CQDs without functional decorating could pass across the BBB. ${ }^{163}$ Polymer-coated nitrogen-doped CQDs (with the sizes in the range of 5-15 nm) which were synthesized by a solvothermal reaction, could enter glioma cells in vitro and be used for in vivo glioma fluorescence imaging (Fig. 11). The physiological pore size upper limit for the bloodtumor barrier (BTB) of malignant glioma microvasculature is around $12 \mathrm{~nm}$ which this implies that the synthesized CQDs can cross the barrier. Furthermore, the hydrophilic polymer coating on the CQDs prolongs blood circulation time and improves the probability of proper targeting at the tumor site. ${ }^{191}$

\subsection{Gene delivery}

Gene therapy which has received considerable attention in biotechnological and medical fields is based on the correction of the origin of diseases through delivery and expression of exogenous DNA encoding for the missing or defective gene product. Therefore, a key factor for gene therapy is the application of proper gene vectors. ${ }^{192}$ Different kinds of nanoparticles ${ }^{193-200}$ and QDs ${ }^{\mathbf{2 0 1 - 2 0 5}}$ have been used for gene delivery. The CQDs could also be used as a platform for gene delivery due to their biocompatibility, low toxicity, strong fluorescence emission, broad excitation spectra, and stable PL. It is shown that the CQDs have a superior capacity to condense plasmid DNA with excellent transfection efficiency. The

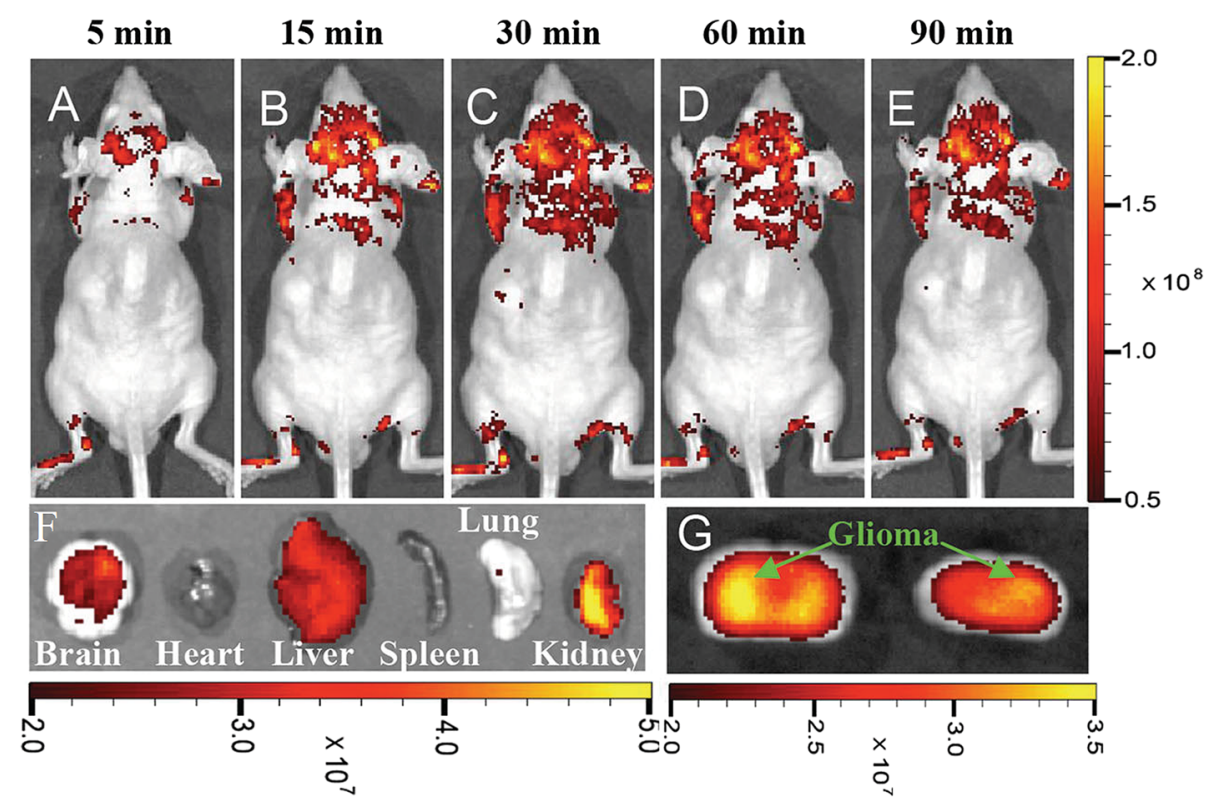

Fig. 11 In vivo and ex vivo imaging of glioma-bearing mice administered with polymer-coated nitrogen-doped CQDs (5-15 nm) intravenously, (A-E) imaging after different post-injection periods, (F) ex vivo imaging of brain, heart, liver, spleen, lung, and kidney $90 \mathrm{~m}$ after CQDs administration, (G) brain coronal imaging 90 min after CQDs administration, reprinted with permission from ref. 191, copyright 2015 John Wiley and Sons. 
cellular uptake of the CQDs/pDNA complexes can be done through both caveolae- and clathrin-mediated endocytosis pathways. ${ }^{206}$ The CQDs with positive surface charge could form a complex with negatively charged siRNAs. It is reported that the folate-conjugated reducible polyethyleneimine passivated CQDs could form a siRNA carrier that releases siRNA in reducing environment. ${ }^{207}$ The CQDS have been used as a gene vector for chondrogenesis from fibroblasts. By application of CQDs, the plasmid SOX9 could be condensed to form nanoparticles in the range of 10-30 nm. The formed nanoparticles have excellent properties such as high solubility, low cytotoxicity and fluorescence emission. ${ }^{\mathbf{1 9 2}}$

\section{Challenges and prospective}

In this review, CQDs were introduced and their optical properties were explained. Different biomedical applications of CQDs such as bioimaging (in vivo and in vitro), drug delivery, cancer therapy, their potential to cross the blood-brain barrier, and gene delivery were discussed.

The heavy metals semiconductor QDs suffer from toxicity and therefore, their biomedical applications should be considered with concerns. However, the CQDs have low toxicity in the human body while having tunable optical properties and fluorescence emission in the NIR region of the light spectrum. Furthermore, the easy synthesis and possibility of functionalizing the CQDs are advantageous over semiconductor QDs. CQDs can be used in versatile biomedical applications such as in vivo and in vitro bioimaging, drug delivery, gene delivery, and cancer therapy. However, more research is needed to evaluate their blood circulation, toxicity, and conjugating them in multifunctional platforms for simultaneous bioimaging and drug/gene delivery. Surface coating and functionalizing, N-doping and route of synthesis will affect optical properties of the CQDs. Therefore, the effect of synthesis parameters and doping should be more investigated in order to reach the desired high QY with biocompatibility. Generally, the CQDs are promising candidates for biomedical applications, instead of semiconductor QDs.

\section{Conflicts of interest}

There are no conflicts of interest to declare.

\section{Acknowledgements}

The author would like to appreciate Shahrood University of Technology and Iranian Nanotechnology Initiative Council for financial support of this project.

\section{References}

$1 \mathrm{X}$. T. Zheng, et al., Glowing graphene quantum dots and carbon dots: properties, syntheses, and biological applications, Small, 2015, 11(14), 1620-1636.

2 M. J. Molaei, A review on nanostructured carbon quantum dots and their applications in biotechnology, sensors, and chemiluminescence, Talanta, 2018, 196, 456-478.
3 P. G. Luo, et al., Carbon-based quantum dots for fluorescence imaging of cells and tissues, RSC Adv., 2014, 4(21), 10791-10807.

4 F. Yuan, et al., Shining carbon dots: synthesis and biomedical and optoelectronic applications, Nano Today, 2016, 11(5), 565-586.

5 P. Mirtchev, et al., Solution phase synthesis of carbon quantum dots as sensitizers for nanocrystalline $\mathrm{TiO}_{2}$ solar cells, J. Mater. Chem., 2012, 22(4), 1265-1269.

6 D. Pan, et al., Hydrothermal route for cutting graphene sheets into blue-luminescent graphene quantum dots, Adv. Mater., 2010, 22(6), 734-738.

7 M. Zhang, et al., Facile synthesis of water-soluble, highly fluorescent graphene quantum dots as a robust biological label for stem cells, J. Mater. Chem., 2012, 22(15), 7461-7467.

8 S. Zhuo, M. Shao and S.-T. Lee, Upconversion and downconversion fluorescent graphene quantum dots: ultrasonic preparation and photocatalysis, ACS Nano, 2012, 6(2), 1059-1064.

$9 \mathrm{~K}$. Welsher, et al., A route to brightly fluorescent carbon nanotubes for near-infrared imaging in mice, Nat. Nanotechnol., 2009, 4(11), 773-780.

10 Q. Mei, et al., Fluorescent graphene oxide logic gates for discrimination of iron (3+) and iron (2+) in living cells by imaging, Chem. Commun., 2012, 48(60), 7468-7470.

$11 \mathrm{~K} . \mathrm{P}$. Loh, et al., Graphene oxide as a chemically tunable platform for optical applications, Nat. Chem., 2010, 2(12), 1015-1024.

12 S.-J. Yu, et al., Bright fluorescent nanodiamonds: no photobleaching and low cytotoxicity, J. Am. Chem. Soc., 2005, 127(50), 17604-17605.

13 J.-P. Boudou, et al., High yield fabrication of fluorescent nanodiamonds, Nanotechnology, 2009, 20(23), 235602.

14 Y.-P. Sun, et al., Quantum-sized carbon dots for bright and colorful photoluminescence, J. Am. Chem. Soc., 2006, 128(24), 7756-7757.

$15 \mathrm{~S}$. Zhu, et al., The photoluminescence mechanism in carbon dots (graphene quantum dots, carbon nanodots, and polymer dots): current state and future perspective, Nano Res., 2015, 8(2), 355-381.

16 M. Bacon, S. J. Bradley and T. Nann, Graphene quantum dots, Part. Part. Syst. Charact., 2014, 31(4), 415-428.

$17 \mathrm{~S}$. Zhu, et al., Strongly green-photoluminescent graphene quantum dots for bioimaging applications, Chem. Commun., 2011, 47(24), 6858-6860.

18 C. Wu and D. T. Chiu, Highly fluorescent semiconducting polymer dots for biology and medicine, Angew. Chem., Int. Ed., 2013, 52(11), 3086-3109.

19 L. Cao, et al., Photoluminescence Properties of Graphene versus Other Carbon Nanomaterials, Acc. Chem. Res., 2013, 46(1), 171-180.

20 C. T. Chien, et al., Tunable photoluminescence from graphene oxide, Angew. Chem., Int. Ed., 2012, 51(27), 6662-6666.

21 O. S. Wolfbeis, An overview of nanoparticles commonly used in fluorescent bioimaging, Chem. Soc. Rev., 2015, 44(14), 4743-4768. 
22 S. Y. Lim, W. Shen and Z. Gao, Carbon quantum dots and their applications, Chem. Soc. Rev., 2015, 44(1), 362-381.

23 L. Cao, et al., Carbon Dots for Multiphoton Bioimaging, J. Am. Chem. Soc., 2007, 129(37), 11318-11319.

$24 \mathrm{H}$. Gonçalves, et al., $\mathrm{Hg}$ (II) sensing based on functionalized carbon dots obtained by direct laser ablation, Sens. Actuators, B, 2010, 145(2), 702-707.

$25 \mathrm{~S}$. Hu, et al., Laser synthesis and size tailor of carbon quantum dots, J. Nanopart. Res., 2011, 13(12), 7247-7252.

$26 \mathrm{~L}$. Zheng, et al., Electrochemiluminescence of WaterSoluble Carbon Nanocrystals Released Electrochemically from Graphite, J. Am. Chem. Soc., 2009, 131(13), 4564-4565.

27 Y.-L. Zhang, et al., Graphitic carbon quantum dots as a fluorescent sensing platform for highly efficient detection of $\mathrm{Fe}^{3+}$ ions, RSC Adv., 2013, 3(11), 3733-3738.

28 L. Wang, et al., Carbon quantum dots displaying dualwavelength photoluminescence and electrochemiluminescence prepared by high-energy ball milling, Carbon, 2015, 94, 472-478.

29 P.-C. Hsu and H.-T. Chang, Synthesis of high-quality carbon nanodots from hydrophilic compounds: role of functional groups, Chem. Commun., 2012, 48(33), 3984-3986.

30 Y. Jiang, et al., A fluorescence turn-off chemosensor based on $\mathrm{N}$-doped carbon quantum dots for detection of $\mathrm{Fe}^{3+}$ in aqueous solution, Mater. Lett., 2015, 141, 366-368.

31 H. Ding, et al., Full-color light-emitting carbon dots with a surface-state-controlled luminescence mechanism, ACS Nano, 2015, 10(1), 484-491.

32 Z. Qian, et al., Highly Luminescent N-Doped Carbon Quantum Dots as an Effective Multifunctional Fluorescence Sensing Platform, Chem.-Eur. J., 2014, 20(8), 2254-2263.

33 S. Mitra, et al., Room temperature and solvothermal green synthesis of self passivated carbon quantum dots, RSC Adv., 2013, 3(10), 3189-3193.

34 A. Rahy, et al., Photoluminescent carbon nanoparticles produced by confined combustion of aromatic compounds, Carbon, 2012, 50(3), 1298-1302.

35 D. Pan, et al., Observation of $\mathrm{pH}^{-}$, solvent-, spin-, and excitation-dependent blue photoluminescence from carbon nanoparticles, Chem. Commun., 2010, 46(21), 3681-3683.

36 R. M. Shereema, et al., One step green synthesis of carbon quantum dots and its application towards the bioelectroanalytical and biolabeling studies, Electrochim. Acta, 2015, 182, 588-595.

37 Y. Liu, et al., One-step microwave-assisted polyol synthesis of green luminescent carbon dots as optical nanoprobes, Carbon, 2014, 68, 258-264.

$38 \mathrm{~J}$. Hou, et al., A novel one-pot route for large-scale preparation of highly photoluminescent carbon quantum dots powders, Nanoscale, 2013, 5(20), 9558-9561.

39 Y. Wang, Q. Zhuang and Y. Ni, Facile Microwave-Assisted Solid-Phase Synthesis of Highly Fluorescent NitrogenSulfur-Codoped Carbon Quantum Dots for Cellular Imaging Applications, Chem.-Eur. J., 2015, 21(37), 1300413011.
40 X. Qin, et al., Microwave-assisted rapid green synthesis of photoluminescent carbon nanodots from flour and their applications for sensitive and selective detection of mercury(II) ions, Sens. Actuators, B, 2013, 184, 156-162.

41 Y. Choi, et al., Microwave-assisted synthesis of luminescent and biocompatible lysine-based carbon quantum dots, $J$. Ind. Eng. Chem., 2017, 47, 329-335.

$42 \mathrm{H}$. Li, et al., Synthesis of fluorescent carbon nanoparticles directly from active carbon via a one-step ultrasonic treatment, Mater. Res. Bull., 2011, 46(1), 147-151.

$43 \mathrm{H}$. Li, et al., Carbon quantum dots $/ \mathrm{Cu}_{2} \mathrm{O}$ composites with protruding nanostructures and their highly efficient (near) infrared photocatalytic behavior, J. Mater. Chem., 2012, 22(34), 17470-17475.

$44 \mathrm{H}$. Li, et al., One-step ultrasonic synthesis of water-soluble carbon nanoparticles with excellent photoluminescent properties, Carbon, 2011, 49(2), 605-609.

45 K. Linehan and H. Doyle, Efficient one-pot synthesis of highly monodisperse carbon quantum dots, RSC Adv., 2014, 4(1), 18-21.

$46 \mathrm{~K}$. Linehan and H. Doyle, Size controlled synthesis of carbon quantum dots using hydride reducing agents, $J$. Mater. Chem. C, 2014, 2(30), 6025-6031.

47 L. Yan, et al., Synthesis of carbon quantum dots by chemical vapor deposition approach for use in polymer solar cell as the electrode buffer layer, Carbon, 2016, 109, 598-607.

$48 \mathrm{~J}$. Wang, et al., 0D/2D interface engineering of carbon quantum dots modified $\mathrm{Bi}_{2} \mathrm{WO}_{6}$ ultrathin nanosheets with enhanced photoactivity for full spectrum light utilization and mechanism insight, Appl. Catal., B, 2018, 222, 115-123.

49 J. Zhang, et al., Highly efficient photocatalysis toward tetracycline of nitrogen doped carbon quantum dots sensitized bismuth tungstate based on interfacial charge transfer, J. Colloid Interface Sci., 2018, 511, 296-306.

50 D. Chen, et al., Preparation of highly sensitive Pt nanoparticles-carbon quantum dots/ionic liquid functionalized graphene oxide nanocomposites and application for $\mathrm{H}_{2} \mathrm{O}_{2}$ detection, Sens. Actuators, B, 2018, 255, 1500-1506.

51 R. Zhang and W. Chen, Nitrogen-doped carbon quantum dots: facile synthesis and application as a "turn-off" fluorescent probe for detection of $\mathrm{Hg}^{2+}$ ions, Biosens. Bioelectron., 2014, 55, 83-90.

52 Z. Lei, et al., Facile synthesis of N-rich carbon quantum dots by spontaneous polymerization and incision of solvents as efficient bioimaging probes and advanced electrocatalysts for oxygen reduction reaction, Nanoscale, 2016, 8(4), 22192226.

$53 \mathrm{M}$. Wu, et al., Preparation of functionalized water-soluble photoluminescent carbon quantum dots from petroleum coke, Carbon, 2014, 78, 480-489.

$54 \mathrm{H}$. Li, et al., Water-soluble fluorescent carbon quantum dots and photocatalyst design, Angew. Chem., Int. Ed., 2010, 49(26), 4430-4434. 
55 Y. Dong, et al., Carbon-based dots co-doped with nitrogen and sulfur for high quantum yield and excitation-independent emission, Angew. Chem., 2013, 125(30), 7954-7958.

56 W. U. Khan, et al., High Quantum Yield Green-Emitting Carbon Dots for Fe(III) Detection, Biocompatible Fluorescent Ink and Cellular Imaging, Sci. Rep., 2017, 1-9.

$57 \mathrm{~J}$. Liu, et al., One-step preparation of nitrogen-doped and surface-passivated carbon quantum dots with high quantum yield and excellent optical properties, $R S C A d v$, 2014, 4(15), 7648.

$58 \mathrm{C}$. Yu, et al., Gadolinium-doped carbon dots with high quantum yield as an effective fluorescence and magnetic resonance bimodal imaging probe, J. Alloys Compd., 2016, 688, 611-619.

59 Y. Dong, et al., Polyamine-functionalized carbon quantum dots for chemical sensing, Carbon, 2012, 50(8), 2810-2815.

$60 \mathrm{M}$. Li, et al., Organic amine-grafted carbon quantum dots with tailored surface and enhanced photoluminescence properties, Carbon, 2015, 91, 291-297.

$61 \mathrm{~J} . \mathrm{H}$. Zhang, et al., In vivo characterization of hair and skin derived carbon quantum dots with high quantum yield as long-term bioprobes in zebrafish, Sci. Rep., 2016, 6(August), 1-12.

62 Q. Liang, et al., Easy synthesis of highly fluorescent carbon quantum dots from gelatin and their luminescent properties and applications, Carbon, 2013, 60, 421-428.

63 M. Yang, et al., N, S co-doped carbon dots with high quantum yield: tunable fluorescence in liquid/solid and extensible applications, J. Nanopart. Res., 2017, 19(6), 217.

64 Z. Y. Yan, et al., Determination of metronidazole by a flowinjection chemiluminescence method using ZnO-doped carbon quantum dots, Xinxing Tan Cailiao, 2014, 29(3), 216-224.

$65 \mathrm{~J}$. Hou, et al., Synthesis and formation mechanistic investigation of nitrogen-doped carbon dots with high quantum yields and yellowish-green fluorescence, Nanoscale, 2016, 8(21), 11185-11193.

66 W. J. Niu, et al., Ethylenediamine-assisted hydrothermal synthesis of nitrogen-doped carbon quantum dots as fluorescent probes for sensitive biosensing and bioimaging, Sens. Actuators, B, 2015, 218, 229-236.

$67 \mathrm{M}$. Li, et al., Solvothermal conversion of coal into nitrogendoped carbon dots with singlet oxygen generation and high quantum yield, Chem. Eng. J., 2017, 320, 570-575.

$68 \mathrm{~W}$. Zhang, et al., Supramolecular interactions via hydrogen bonding contributing to citric-acid derived carbon dots with high quantum yield and sensitive photoluminescence, RSC Adv., 2017, 7(33), 20345-20353.

69 Y. Dong, et al., Extraction of electrochemiluminescent oxidized carbon quantum dots from activated carbon, Chem. Mater., 2010, 22(21), 5895-5899.

$70 \mathrm{~S}$. Zhu, et al., Highly photoluminescent carbon dots for multicolor patterning, sensors, and bioimaging, Angew. Chem., Int. Ed., 2013, 52(14), 3953-3957.
$71 \mathrm{~J}$. Yu, et al., Green preparation of carbon dots by Jinhua bergamot for sensitive and selective fluorescent detection of $\mathrm{Hg}^{2+}$ and $\mathrm{Fe}^{3+}$, Sens. Actuators, B, 2015, 214(3), 29-35.

72 J. Niu and H. Gao, Synthesis and drug detection performance of nitrogen-doped carbon dots, J. Lumin., 2014, 149, 159-162.

73 P. Pierrat, et al., Efficient in vitro and in vivo pulmonary delivery of nucleic acid by carbon dot-based nanocarriers, Biomaterials, 2015, 51, 290-302.

74 Z. H. Gao, et al., Carbon dots-based fluorescent probe for trace $\mathrm{Hg}^{2+}$ detection in water sample, Sens. Actuators, B, 2016, 222, 965-971.

75 S. Dong, et al., Rapid Screening of Oxygen-States in Carbon Quantum Dots by Chemiluminescence Probe Rapid Screening of Oxygen-States in Carbon Quantum Dots by Chemiluminescence Probe, Anal. Chem., 2017, 89(22), 12520-12526.

$76 \mathrm{H}$. Su, Facile synthesis of N-rich carbon quantum dots from porphyrins as efficient probes for bioimaging and biosensing in living cells, Int. J. Nanomed., 2017, 12, 73757391.

77 F. Niu, et al., Controllable electrochemical/electroanalytical approach to generate nitrogen-doped carbon quantum dots from varied amino acids: pinpointing the utmost quantum yield and the versatile photoluminescent and electrochemiluminescent applications, Electrochim. Acta, 2017, 236, 239-251.

78 S. L. D'souza, et al., Synthesis of fluorescent carbon dots using Daucus carota subsp. sativus roots for mitomycin drug delivery, Optik, 2018, 158, 893-900.

79 A. Kumari, et al., Synthesis of green fluorescent carbon quantum dots using waste polyolefins residue for $\mathrm{Cu}^{2+}$ ion sensing and live cell imaging, Sens. Actuators, B, 2018, 254, 197-205.

$80 \mathrm{~W}$. Kwon, et al., Freestanding luminescent films of nitrogen-rich carbon nanodots toward large-scale phosphor-based white-light-emitting devices, Chem. Mater., 2013, 25(9), 1893-1899.

81 Y.-Y. Yao, et al., Magnetofluorescent carbon dots derived from crab shell for targeted dual-modality bioimaging and drug delivery, ACS Appl. Mater. Interfaces, 2017, 9(16), 13887-13899.

82 B. A. Kairdolf, et al., Semiconductor quantum dots for bioimaging and biodiagnostic applications, Annu. Rev. Anal. Chem., 2013, 6, 143-162.

83 C. Manzo and M. F. Garcia-Parajo, A review of progress in single particle tracking: from methods to biophysical insights, Rep. Prog. Phys., 2015, 78(12), 124601.

$84 \mathrm{~J}$. Li and J.-J. Zhu, Quantum dots for fluorescent biosensing and bio-imaging applications, Analyst, 2013, 138(9), 25062515.

85 P. Zrazhevskiy, M. Sena and X. Gao, Designing multifunctional quantum dots for bioimaging, detection, and drug delivery, Chem. Soc. Rev., 2010, 39(11), 4326-4354.

86 D. V. Freitas, et al., Toward greener electrochemical synthesis of composition-tunable luminescent CdX-based 
$(\mathrm{X}=\mathrm{Te}, \mathrm{Se}, \mathrm{S})$ quantum dots for bioimaging cancer cells, Sens. Actuators, B, 2017, 250, 233-243.

87 K. Shivaji, et al., Green-Synthesis-Derived CdS Quantum Dots Using Tea Leaf Extract: Antimicrobial, Bioimaging, and Therapeutic Applications in Lung Cancer Cells, ACS Appl. Nano Mater., 2018, 1(4), 1683-1693.

88 S. de Carvalho, et al., In vitro and in vivo assessment of nanotoxicity of $\mathrm{CdS}$ quantum dot/aminopolysaccharide bionanoconjugates, Mater. Sci. Eng., C, 2017, 71, 412-424.

89 L. Chen, et al., Aqueous synthesis and biostabilization of CdS@ZnS quantum dots for bioimaging applications, Mater. Res. Express, 2015, 2(10), 105401.

$90 \mathrm{~J}$. Wang, et al., Ratiometric ultrasensitive fluorometric detection of ascorbic acid using a dually emitting CdSe@SiO ${ }_{2} @ C d T e$ quantum dot hybrid, Microchim. Acta, 2018, 185(1), 42.

91 A. Kumari, et al., Proficient surface modification of CdSe quantum dots for highly luminescent and biocompatible probes for bioimaging: a comparative experimental investigation, J. Lumin., 2018, 199, 174-182.

92 L. Maestro, et al., Deep tissue bio-imaging using twophoton excited CdTe fluorescent quantum dots working within the biological window, Nanoscale, 2012, 4(1), 298302.

93 S. Chakraborty, et al., Multifunctional, high luminescent, biocompatible CdTe quantum dot fluorophores for bioimaging applications, Int. J. Nanosci., 2011, 10(04n05), 1191-1195.

94 M. V. Bharathi, et al., Water-mediated green synthesis of $\mathrm{PbS}$ quantum dot and its glutathione and biotin conjugates for non-invasive live cell imaging, $R$. Soc. Open Sci., 2018, 5(3), 171614.

$95 \mathrm{~S} . \mathrm{Xu}, \mathrm{D} . \mathrm{Li}$ and $\mathrm{P}$. Wu, One-pot, facile, and versatile synthesis of monolayer $\mathrm{MoS}_{2} / \mathrm{WS}_{2}$ quantum dots as bioimaging probes and efficient electrocatalysts for hydrogen evolution reaction, Adv. Funct. Mater., 2015, 25(7), 1127-1136.

96 J. Wang, et al., $\mathrm{MoS}_{2}$ quantum dot@polyaniline inorganicorganic nanohybrids for in vivo dual-modal imaging guided synergistic photothermal/radiation therapy, ACS Appl. Mater. Interfaces, 2016, 8(37), 24331-24338.

97 W. Dai, et al., Tunable fabrication of molybdenum disulfide quantum dots for intracellular microRNA detection and multiphoton bioimaging, Small, 2015, 11(33), 4158-4164.

98 H. Lin, et al., Colloidal synthesis of $\mathrm{MoS}_{2}$ quantum dots: size-dependent tunable photoluminescence and bioimaging, New J. Chem., 2015, 39(11), 8492-8497.

$99 \mathrm{~K}$. Zhou, et al., As-prepared $\mathrm{MoS}_{2}$ quantum dot as a facile fluorescent probe for long-term tracing of live cells, Nanotechnology, 2016, 27(27), 275101.

100 B. Mahler, et al., Towards non-blinking colloidal quantum dots, Nat. Mater., 2008, 7(8), 659-664.

101 A. Dehghani, et al., Collagen derived carbon quantum dots for cell imaging in 3D scaffolds via two-photon spectroscopy, Carbon, 2018, 131, 238-245.
102 S.-H. Chiu, et al., Rapid fabrication of carbon quantum dots as multifunctional nanovehicles for dual-modal targeted imaging and chemotherapy, Acta Biomater., 2016, 46, 151-164.

103 N. Vasimalai, et al., Green synthesis of fluorescent carbon dots from spices for in vitro imaging and tumour cell growth inhibition, Beilstein J. Nanotechnol., 2018, 9, 530.

104 V. Ramanan, et al., Outright green synthesis of fluorescent carbon dots from eutrophic algal blooms for in vitro imaging, ACS Sustainable Chem. Eng., 2016, 4(9), 47244731.

$105 \mathrm{~J}$. Yang, et al., Ultrasmall and photostable nanotheranostic agents based on carbon quantum dots passivated with polyamine-containing organosilane molecules, Nanoscale, 2017, 9(40), 15441-15452.

106 G. Gao, et al., On-off-on fluorescent nanosensor for $\mathrm{Fe}^{3+}$ detection and cancer/normal cell differentiation via silicon-doped carbon quantum dots, Carbon, 2018, 134, 232-243.

107 S. Aiyer, et al., Fluorescent carbon nanodots for targeted in vitro cancer cell imaging, Appl. Mater. Today, 2016, 4, 71-77.

108 L.-P. Guo, Y. Zhang and W.-C. Li, Sustainable microalgae for the simultaneous synthesis of carbon quantum dots for cellular imaging and porous carbon for $\mathrm{CO}_{2}$ capture, J. Colloid Interface Sci., 2017, 493, 257-264.

109 A. K. Samantara, et al., Good's buffer derived highly emissive carbon quantum dots: excellent biocompatible anticancer drug carrier, J. Mater. Chem. B, 2016, 4(14), 2412-2420.

110 Z. Liu, et al., Carbon-Quantum-Dots-Loaded Mesoporous Silica Nanocarriers with $\mathrm{pH}$-Switchable Zwitterionic Surface and Enzyme-Responsive Pore-Cap for Targeted Imaging and Drug Delivery to Tumor, Adv. Healthcare Mater., 2016, 5(12), 1401-1407.

111 M. Zhang, et al., Near-infrared light triggered phototherapy, in combination with chemotherapy using magnetofluorescent carbon quantum dots for effective cancer treating, Carbon, 2017, 118, 752-764.

112 A. Kundu, et al., Facile and green approach to prepare fluorescent carbon dots: emergent nanomaterial for cell imaging and detection of vitamin B2, J. Colloid Interface Sci., 2016, 468, 276-283.

$113 \mathrm{X}$. Chen, et al., Highly cysteine-selective fluorescent nanoprobes based on ultrabright and directly synthesized carbon quantum dots, Anal. Bioanal. Chem., 2018, 410(12), 2961-2970.

114 S.-T. Yang, et al., Carbon dots as nontoxic and highperformance fluorescence imaging agents, J. Phys. Chem. C, 2009, 113(42), 18110-18114.

115 A. Galeone, et al., In vivo assessment of CdSe-ZnS quantum dots: coating dependent bioaccumulation and genotoxicity, Nanoscale, 2012, 4(20), 6401-6407.

116 S. Bozrova, et al., In Vitro Cytotoxicity of CdSe/ZnS Quantum Dots and Their Interaction with Biological Systems, KnE Energy \& Physics, 2018, 3(2), 58-63.

117 S. Chinnathambi, N. Abu and N. Hanagata, Biocompatible $\mathrm{CdSe} / \mathrm{ZnS}$ quantum dot micelles for long-term cell imaging without alteration to the native structure of the blood 
plasma protein human serum albumin, $R S C A d v ., 2017$, 7(5), 2392-2402.

118 B.-T. Lee, et al., Bioaccumulation and in vivo dissolution of CdSe/ZnS with three different surface coatings by Daphnia magna, Chemosphere, 2016, 143, 115-122.

119 G. Xu, et al., The Reproductive Toxicity of CdSe/ZnS Quantum Dots on the in vivo Ovarian Function and in vitro Fertilization, Sci. Rep., 2016, 6, 37677.

120 Y. Lv, et al., Highly sensitive and accurate detection of Creactive protein by $\mathrm{CdSe} / \mathrm{ZnS}$ quantum dot-based fluorescence-linked immunosorbent assay, $J$. Nanobiotechnol., 2017, 15(1), 35.

$121 \mathrm{~J}$. Chen, et al., Hybrid nanoprobes of bismuth sulfide nanoparticles and $\mathrm{CdSe} / \mathrm{ZnS}$ quantum dots for mouse computed tomography/fluorescence dual mode imaging, J. Nanobiotechnol., 2015, 13(1), 76.

122 Y.-Y. Zhang, et al., A new hydrothermal refluxing route to strong fluorescent carbon dots and its application as fluorescent imaging agent, Talanta, 2013, 117, 196-202.

123 S. Ray, et al., Fluorescent carbon nanoparticles: synthesis, characterization, and bioimaging application, J. Phys. Chem. C, 2009, 113(43), 18546-18551.

124 Y. Xu, et al., Nitrogen-Doped Carbon Dots: A Facile and General Preparation Method, Photoluminescence Investigation, and Imaging Applications, Chem.-Eur. J., 2013, 19(7), 2276-2283.

125 G. Eda, et al., Blue photoluminescence from chemically derived graphene oxide, Adv. Mater., 2010, 22(4), 505-509.

$126 \mathrm{~L}$. $\mathrm{Hu}$, et al., Multifunctional carbon dots with high quantum yield for imaging and gene delivery, Carbon, 2014, 67, 508-513.

127 W. Li, et al., Simple and Green Synthesis of Nitrogen-Doped Photoluminescent Carbonaceous Nanospheres for Bioimaging, Angew. Chem., Int. Ed., 2013, 52(31), 81518155.

128 Y. Fang, et al., Easy synthesis and imaging applications of cross-linked green fluorescent hollow carbon nanoparticles, ACS Nano, 2011, 6(1), 400-409.

129 Z.-A. Qiao, et al., Commercially activated carbon as the source for producing multicolor photoluminescent carbon dots by chemical oxidation, Chem. Commun., 2010, 46(46), 8812-8814.

130 W.-S. Zou, et al., Insecticide as a precursor to prepare highly bright carbon dots for patterns printing and bioimaging: a new pathway for making poison profitable, Chem. Eng. J., 2016, 294, 323-332.

131 D. Kumar, et al., Synthesis and characterization of carbon quantum dots from orange juice, J. Bionanosci., 2014, 8(4), 274-279.

132 V. N. Mehta, et al., One-step hydrothermal approach to fabricate carbon dots from apple juice for imaging of mycobacterium and fungal cells, Sens. Actuators, B, 2015, 213, 434-443.

$133 \mathrm{Y}$. $\mathrm{Xu}$, et al., Green Synthesis of Fluorescent Carbon Quantum Dots for Detection of $\mathrm{Hg}^{2+}$, Chin. J. Anal. Chem., 2014, 42(9), 1252-1258.
134 A.-M. Alam, et al., Synthesis of carbon quantum dots from cabbage with down-and up-conversion photoluminescence properties: excellent imaging agent for biomedical applications, Green Chem., 2015, 17(7), 3791-3797.

135 B. S. B. Kasibabu, et al., Imaging of bacterial and fungal cells using fluorescent carbon dots prepared from carica papaya juice, J. Fluoresc., 2015, 25(4), 803-810.

136 B. De and N. Karak, A green and facile approach for the synthesis of water soluble fluorescent carbon dots from banana juice, $R S C A d v$., 2013, 3(22), 8286-8290.

137 R. Atchudan, T. N. J. I. Edison and Y. R. Lee, Nitrogendoped carbon dots originating from unripe peach for fluorescent bioimaging and electrocatalytic oxygen reduction reaction, J. Colloid Interface Sci., 2016, 482, 8-18.

$138 \mathrm{Y}$. Hu, et al., Waste frying oil as a precursor for one-step synthesis of sulfur-doped carbon dots with $\mathrm{pH}$-sensitive photoluminescence, Carbon, 2014, 77, 775-782.

139 L. Wang and H. S. Zhou, Green synthesis of luminescent nitrogen-doped carbon dots from milk and its imaging application, Anal. Chem., 2014, 86(18), 8902-8905.

140 C. Zhu, J. Zhai and S. Dong, Bifunctional fluorescent carbon nanodots: green synthesis via soy milk and application as metal-free electrocatalysts for oxygen reduction, Chem. Commun., 2012, 48(75), 9367-9369.

$141 \mathrm{X}$. Yang, et al., Novel and green synthesis of highfluorescent carbon dots originated from honey for sensing and imaging, Biosens. Bioelectron., 2014, 60, 292298.

142 J. Wang, C. F. Wang and S. Chen, Amphiphilic egg-derived carbon dots: rapid plasma fabrication, pyrolysis process, and multicolor printing patterns, Angew. Chem., Int. Ed., 2012, 51(37), 9297-9301.

143 P.-C. Hsu, et al., Synthesis and analytical applications of photoluminescent carbon nanodots, Green Chem., 2012, 14(4), 917-920.

144 S. Thambiraj and R. Shankaran, Green synthesis of highly fluorescent carbon quantum dots from sugarcane bagasse pulp, Appl. Surf. Sci., 2016, 390, 435-443.

145 V. N. Mehta, S. Jha and S. K. Kailasa, One-pot green synthesis of carbon dots by using Saccharum officinarum juice for fluorescent imaging of bacteria (Escherichia coli) and yeast (Saccharomyces cerevisiae) cells, Mater. Sci. Eng., C, 2014, 38, 20-27.

146 S. Sahu, et al., Simple one-step synthesis of highly luminescent carbon dots from orange juice: application as excellent bio-imaging agents, Chem. Commun., 2012, 48(70), 8835-8837.

147 Z. Qian, et al., Highly Luminescent N-Doped Carbon Quantum Dots as an Effective Multifunctional Fluorescence Sensing Platform, Chem.-Eur. J., 2014, 20(8), 2254-2263.

$148 \mathrm{H}$. Tao, et al., In vivo NIR fluorescence imaging, biodistribution, and toxicology of photoluminescent carbon dots produced from carbon nanotubes and graphite, Small, 2012, 8(2), 281-290. 
$149 \mathrm{~J} . \mathrm{Ge}$, et al., Red-emissive carbon dots for fluorescent, photoacoustic, and thermal theranostics in living mice, Adv. Mater., 2015, 27(28), 4169-4177.

150 B. Kong, et al., Carbon dot-based inorganic-organic nanosystem for two-photon imaging and biosensing of $\mathrm{pH}$ variation in living cells and tissues, Adv. Mater., 2012, 24(43), 5844-5848.

151 Q. Zhan, et al., Controlling the excitation of upconverting luminescence for biomedical theranostics: neodymium sensitizing, Opt. Mater. Express, 2016, 6(4), 1011-1023.

152 X. Jia, J. Li and E. Wang, One-pot green synthesis of optically $\mathrm{pH}$-sensitive carbon dots with upconversion luminescence, Nanoscale, 2012, 4(18), 5572-5575.

$153 \mathrm{~J}$. Shen, et al., Facile preparation and upconversion luminescence of graphene quantum dots, Chem. Commun., 2011, 47(9), 2580-2582.

$154 \mathrm{~K}$. Jiang, et al., Red, green, and blue luminescence by carbon dots: full-color emission tuning and multicolor cellular imaging, Angew. Chem., Int. Ed., 2015, 54(18), 5360-5363.

155 S. K. Bhunia, et al., Carbon nanoparticle-based fluorescent bioimaging probes, Sci. Rep., 2013, 3, 1473.

156 C. Liu, et al., Nano-carrier for gene delivery and bioimaging based on carbon dots with PEI-passivation enhanced fluorescence, Biomaterials, 2012, 33(13), 3604-3613.

157 N. Parvin and T. K. Mandal, Synthesis of a highly fluorescence nitrogen-doped carbon quantum dots bioimaging probe and its in vivo clearance and printing applications, RSC Adv., 2016, 6(22), 18134-18140.

$158 \mathrm{~J}$. Wang, et al., Synthesis, characterization and cells and tissues imaging of carbon quantum dots, Opt. Mater., 2017, 72, 15-19.

159 C. T. Matea, et al., Quantum dots in imaging, drug delivery and sensor applications, Int. J. Nanomed., 2017, 12, 5421.

$160 \mathrm{~J}$. Jana, et al., One pot synthesis of intriguing fluorescent carbon dots for sensing and live cell imaging, Talanta, 2016, 150, 253-264.

161 G. E. Gu, et al., Fluorescent polydopamine nanoparticles as a probe for zebrafish sensory hair cells targeted in vivo imaging, Sci. Rep., 2018, 8(1), 4393.

$162 \mathrm{~L}$. Wu, et al., Surface passivation of carbon nanoparticles with branched macromolecules influences near infrared bioimaging, Theranostics, 2013, 3(9), 677.

$163 \mathrm{~N}$. Li, et al., Biodistribution study of carbogenic dots in cells and in vivo for optical imaging, J. Nanopart. Res., 2012, 14(10), 1177.

164 S. Srivastava, et al., Magnetic-nanoparticle-doped carbogenic nanocomposite: an effective magnetic resonance/fluorescence multimodal imaging probe, Small, 2012, 8(7), 1099-1109.

165 S.-T. Yang, et al., Carbon dots for optical imaging in vivo, J. Am. Chem. Soc., 2009, 131(32), 11308-11309.

$166 \mathrm{~L}$. Nie and X. Chen, Structural and functional photoacoustic molecular tomography aided by emerging contrast agents, Chem. Soc. Rev., 2014, 43(20), 7132-7170.
167 M. S. Khan, et al., Controlled delivery of dopamine hydrochloride using surface modified carbon dots for neuro diseases, Colloids Surf., B, 2015, 134, 140-146.

168 O. C. Farokhzad and R. Langer, Impact of nanotechnology on drug delivery, ACS Nano, 2009, 3(1), 16-20.

169 Z. Liu, et al., Targeted Drug Delivery: Carbon-Quantum-Dots-Loaded Mesoporous Silica Nanocarriers with $\mathrm{pH}$-Switchable Zwitterionic Surface and Enzyme-Responsive Pore-Cap for Targeted Imaging and Drug Delivery to Tumor, Adv. Healthcare Mater., 2016, 5(12), 1380.

170 T. Feng, et al., Dual-responsive carbon dots for tumor extracellular microenvironment triggered targeting and enhanced anticancer drug delivery, ACS Appl. Mater. Interfaces, 2016, 8(29), 18732-18740.

171 A. R. Chowdhuri, et al., Carbon dots embedded magnetic nanoparticles@chitosan@metal organic framework as a nanoprobe for $\mathrm{pH}$ sensitive targeted anticancer drug delivery, ACS Appl. Mater. Interfaces, 2016, 8(26), 1657316583.

172 Z. Peng, et al., Carbon dots: promising biomaterials for bone-specific imaging and drug delivery, Nanoscale, 2017, 9(44), 17533-17543.

173 S. Karthik, et al., Photoresponsive quinoline tethered fluorescent carbon dots for regulated anticancer drug delivery, Chem. Commun., 2013, 49(89), 10471-10473.

$174 \mathrm{~J}$. Yang, et al., Fabrication and characterization of hollow starch nanoparticles by gelation process for drug delivery application, Carbohydr. Polym., 2017, 173, 223-232.

$175 \mathrm{C}$. $\mathrm{Xu}$, et al., Magnetically targeted co-delivery of hydrophilic and hydrophobic drugs with hollow mesoporous ferrite nanoparticles, $R S C A d v$., 2018, 8(28), 15326-15335.

$176 \mathrm{~J}$. Yang, et al., Multifunctional zinc-based hollow nanoplatforms as a smart $\mathrm{pH}$-responsive drug delivery system to enhance in vivo tumor-inhibition efficacy, Mater. Des., 2018, 139, 172-180.

177 Q. Wang, et al., Hollow luminescent carbon dots for drug delivery, Carbon, 2013, 59, 192-199.

178 Y. Shu, et al., Ionic liquid mediated organophilic carbon dots for drug delivery and bioimaging, Carbon, 2017, 114, 324-333.

179 V. N. Mehta, et al., Green synthetic approach for synthesis of fluorescent carbon dots for lisinopril drug delivery system and their confirmations in the cells, J. Fluoresc., 2017, 27(1), 111-124.

180 N. Sarkar, et al., Carbon quantum dot tailored calcium alginate hydrogel for $\mathrm{pH}$ responsive controlled delivery of vancomycin, Eur. J. Pharm. Sci., 2017, 109, 359-371.

181 Y. Wang, et al., Fluorescent carbon dot-gated multifunctional mesoporous silica nanocarriers for redox/ enzyme dual-responsive targeted and controlled drug delivery and real-time bioimaging, Eur. J. Pharm. Biopharm., 2017, 117, 105-115.

182 S. M. Ardekani, et al., Two-photon excitation triggers combined chemo-photothermal therapy via doped carbon 
nanohybrid dots for effective breast cancer treatment, Chem. Eng. J., 2017, 330, 651-662.

183 B. Ma, et al., Prolonged fluorescence lifetime of carbon quantum dots by combining with hydroxyapatite nanorods for bio-applications, Nanoscale, 2017, 9(6), 2162-2171.

184 M. Zhang, et al., pH-Sensitive N-doped carbon dotsheparin and doxorubicin drug delivery system: preparation and anticancer research, RSC Adv., 2017, 7(15), 9347-9356.

185 M. Zhang, et al., Magnetofluorescent $\mathrm{Fe}_{3} \mathrm{O}_{4} /$ carbon quantum dots coated single-walled carbon nanotubes as dual-modal targeted imaging and chemo/photodynamic/ photothermal triple-modal therapeutic agents, Chem. Eng. J., 2018, 338, 526-538.

186 R. K. Das, et al., Magnetic Mesoporous Silica Gated with Doped Carbon Dot for Site-Specific Drug Delivery, Fluorescence, and MR Imaging, Langmuir, 2018, 34(18), 5253-5262.

187 N. Huang, et al., Efficacy of NGR peptide-modified PEGylated quantum dots for crossing the blood-brain barrier and targeted fluorescence imaging of glioma and tumor vasculature, Nanomedicine, 2017, 13(1), 83-93.

188 M. M. Patel and B. M. Patel, Crossing the blood-brain barrier: recent advances in drug delivery to the brain, CNS Drugs, 2017, 31(2), 109-133.

189 J. Gu, K. Al-Bayati and E. A. Ho, Development of antibodymodified chitosan nanoparticles for the targeted delivery of siRNA across the blood-brain barrier as a strategy for inhibiting HIV replication in astrocytes, Drug Delivery Transl. Res., 2017, 7(4), 497-506.

190 A. V. Kabanov and E. V. Batrakova, Polymer nanomaterials for drug delivery across the blood brain barrier, in Neuroimmune Pharmacology, 2017, Springer, p. 847-868.

191 Y. Wang, et al., Direct Solvent-Derived Polymer-Coated Nitrogen-Doped Carbon Nanodots with High Water Solubility for Targeted Fluorescence Imaging of Glioma, Small, 2015, 11(29), 3575-3581.

192 X. Cao, et al., Photoluminescent Cationic Carbon Dots as efficient Non-Viral Delivery of Plasmid SOX9 and Chondrogenesis of Fibroblasts, Sci. Rep., 2018, 8(1), 7057.

193 M. S. Huh, et al., Polysaccharide-based nanoparticles for gene delivery, Top. Curr. Chem., 2017, 375(2), 31.

$194 \mathrm{~J}$. Kim, et al., Gene delivery nanoparticles to modulate angiogenesis, Adv. Drug Delivery Rev., 2017, 119, 20-43.
195 I. M. El-Sherbiny, et al., Magnetic nanoparticles-based drug and gene delivery systems for the treatment of pulmonary diseases, Nanomedicine, 2017, 12(4), 387-402.

196 I. Fernandez-Piñeiro, et al., Development and characterisation of chondroitin sulfate-and hyaluronic acid-incorporated sorbitan ester nanoparticles as gene delivery systems, Eur. J. Pharm. Biopharm., 2018, 125, 85-94.

197 X. Huang, et al., Characterization of calcium phosphate nanoparticles based on a PEGylated chelator for gene delivery, ACS Appl. Mater. Interfaces, 2017, 9(12), 1043510445.

198 B. Du, et al., Lipid-Coated Gold Nanoparticles Functionalized by Folic Acid as Gene Vectors for Targeted Gene Delivery in vitro and in vivo, ChemMedChem, 2017, 12(21), 1768-1775.

199 M. C. Kim, et al., Polyethyleneimine-associated polycaprolactone-Superparamagnetic iron oxide nanoparticles as a gene delivery vector, J. Biomed. Mater. Res., Part B, 2017, 105(1), 145-154.

200 P. Zhang, et al., Regulation the morphology of cationized gold nanoparticles for effective gene delivery, Colloids Surf., B, 2017, 157, 18-25.

201 M. V. Yezhelyev, et al., Proton-sponge coated quantum dots for siRNA delivery and intracellular imaging, J. Am. Chem. Soc., 2008, 130(28), 9006-9012.

202 S. M. Ghafary, et al., Simultaneous Gene Delivery and Tracking through Preparation of Photo-Luminescent Nanoparticles Based on Graphene Quantum Dots and Chimeric Peptides, Sci. Rep., 2017, 7(1), 9552.

203 G. Lin, et al., Quantum dots-siRNA nanoplexes for gene silencing in central nervous system tumor cells, Front. Pharmacol., 2017, 8, 182.

204 F. Yin, et al., Black phosphorus quantum dot based novel siRNA delivery systems in human pluripotent teratoma PA-1 cells, J. Mater. Chem. B, 2017, 5(27), 5433-5440.

$205 \mathrm{X}$. Yang, et al., One-step synthesis of photoluminescent carbon dots with excitation-independent emission for selective bioimaging and gene delivery, J. Colloid Interface Sci., 2017, 492, 1-7.

$206 \mathrm{~J}$. Zhou, et al., Cationic carbon quantum dots derived from alginate for gene delivery: one-step synthesis and cellular uptake, Acta Biomater., 2016, 42, 209-219.

207 Y.-F. Wu, et al., Multi-functionalized carbon dots as theranostic nanoagent for gene delivery in lung cancer therapy, Sci. Rep., 2016, 6, 21170. 